ISSN: 2162-3104 Print/ ISSN: 2166-3750 Online

Volume 8, Issue 4 (2018), pp. 1783-1814

(C) Journal of International Students

http://jistudents.org/

doi:10.5281/zenodo. 1469857

\title{
Examining the Sexual Enigma of the Immigrant Paradox with International Students
}

\author{
Philip A. Belcastro \\ Hardaye Ramsaroop-Hansen \\ The City University of New York Borough of Manhattan Community College, USA
}

\begin{abstract}
The metamorphosis of core sexual practices and sexual orientation norms resulting from the acculturation of U.S. native and non-native residents remains an enigma. A convenience sample from a metropolitan community college was drawn to describe the consequences of acculturation on U.S. international students' core sexual practices and sexual orientation. Findings include: (1) observed differences in male international student sexual practices to that of the native male population were attributed to social opportunity and not the immigrant paradox, and (2) female international students' core sexual practices and sexual orientation shifted over time to the higher risk sexual practices of the native female population. The U.S. needs to commit resources including qualified health educators, to support state mandated public school and undergraduate sex education for U.S. juveniles and young adults.
\end{abstract}

Keywords: immigrant paradox, sexual practices, sexual orientation, sex education, international student

Graves (1967) identified two levels of acculturation: (1) the individual level, being the psychological change within the individual resulting from contact with the host society, and (2) the group level, being the collective process in which there is change in either the native culture or the merging culture or both. The engine of acculturation is cultural diffusion. Cultural diffusion is time dependent. Increased exposure of commingling cultures over time can reshape individual behavior (individual level). Increased scale of exposure within commingling cultures can reshape cultural norms (group level). The reshaping of individuals and groups by acculturation is not necessarily unidirectional, that is exclusively from the host culture to the merging culture. The metamorphosis of core sexual practices and sexual orientation norms resulting from the acculturation of U.S. native and non-native residents remains an enigma.

\section{LITERATURE REVIEW}

Researchers examining the effects of acculturation in the U.S. report that first generation immigrants had comparable or superior morbidity and mortality health metrics than that of native Whites (Coleman-Minahan, Chavez, \& Bull, 2017; Guilamo-Ramos, Jaccard, Pena, \& 
Goldberg, 2005; Lopez-Gonzalez, Aravena, \& Hummer, 2005). Researchers referred to this unanticipated observation as the 'epidemiological paradox' (Marks, Ejesi, \& Garcia Coll, 2014). Detractors have argued that the epidemiological paradox is an anomaly of research design resulting from sampling error. Regardless, a body of literature continues to support the epidemiological paradox and its corollary, the 'immigrant paradox', as well as its derivative the 'Hispanic paradox' (Guarini, Marks, Patton, \& Garcia Coll, 2013; Schwartz, et al., 2014; Teruya, \& Bazargan-Hejazi, 2013).

In tandem with the epidemiological paradox, the immigrant paradox purports that first generation immigrants exhibit 'more favorable sexual practices' and subsequently health outcomes than second or third generation immigrants having greater acculturation exposure (Garcia Coll, \& Marks, 2012; Guarini, Marks, Patton, \& Garcia Coll, 2011; Parrado, \& Flippen, 2010). By example, in a survey examining HIV risk behaviors, Hispanic immigrants reported decreased condom use relative to time spent in the U.S. (Sastre, Sanchez, \& De La Rosa, 2015). A cross-sectional HIV risk assessment of Mexican immigrants returning to Mexico from the U.S. revealed increased homosexual encounters, substance abuse before coitus, decreased condom use, increased casual coital partners, and increased frequency in hiring sex workers (Zhang, et al., 2017). A study of Hispanic men and non-Hispanic White men living in the U.S. reported mixed results regarding their frequency of sexual activity with sex workers as well as their number of lifetime sexual partners (Haderxhanaj, Rhodes, Romaguera, Bloom, \& Leichliter, 2015).

An inherent design flaw in these investigations is the premise that increased frequency in select high-risk sexual behaviors such as hiring sex workers and lifetime coital partners is a benchmark of acculturation. Equating high-risk sexual behaviors (i.e. condom usage, number of lifetime sexual partners, and contact with sex workers) as evidence of a cultural shift in immigrants' indigenous sexual norms disregards the accessibility to and availability of sexual practices by the host culture. Positing that such limited high-risk sexual practices are representative of an acculturated shift in norms ignores the fact that sexual practices, sexual relationships, and procreation are fundamental commonalities of human behavior and inherently encompass cultural overlap - albeit personalized by cultural idiosyncrasy.

Accordingly, acculturation studies report conflicting observations when examining the effects of acculturation on sexual practices ( $\mathrm{Du}, \& \mathrm{Li}, 2015)$. By example, a survey of 2,635 adolescent immigrants residing in Massachusetts for less than six years reported that adolescents had less lifetime substance use than students who lived in the U.S. their entire lives. However, the same study yielded similar rates of sexual intercourse (Blake, Ledsky, Goodenow, \& O'Donnell, 2001). Montealegre, Risser, Selwyn, Mccurdy, and Sabin (2012) compared immigrants with less than five years residency in the U.S. to those with more than five years, and reported that the latter was more likely to be in a stable sexual relationship, with less lifetime sexual partners and longer periods of relationship duration.

Representing a limited number of sexual behaviors as evidence of a cultural shift in a population's core sexual and procreation norms is problematic. It is the core sexual and procreation practices of a community melded to their sexual orientation that comprises cultural norms. If the immigrant population's core sexual practices are similar to that of the host upon introduction to the community--then changes in behavior may be adaptive or opportunistic rather than adoptive or evidence of an acculturated shift.

Nationwide surveys present another threat to verifying the immigrant paradox. While U.S. surveys such as the National Health and Social Life Survey, National Surveys of Sexual Health and Behavior, and Sexual Exploration in America Study account for race and ethnicity, in 
general they do not account for distinctions between immigrant populations and native populations (Herbenick, et al., 2017). Such surveys are represented as native population norms, when in fact U.S. native populations are indeterminately blended with U.S. immigrant populations. In addition, national surveys by design and economics are limited in their scope of sexual practices queried.

Descriptive research that accounts for the effects of acculturative exposure on U.S. immigrant populations' collective core of sexual practices, that is masturbation, oral-genital stimulation, coitus, and procreation--including taboo sexual practices is noticeably absent from the literature. Furthermore, sexual orientation is a cornerstone of the human sexual experience and cannot be omitted when assessing cultural shifts in sexual practice norms of a population. The challenge is to determine whether the engine of acculturation, which is cultural diffusion, over time is reshaping the sexual and procreational practices as well as sexual orientation of commingling cultures.

International undergraduates, as in U.S. immigrant populations, have U.S. non-citizen, resident status. International undergraduates, as in U.S. immigrant populations, experience acculturative exposure for varying lengths of time. As such, there should be evidence of the immigrant paradox in international student populations' sexual orientation and core sexual and procreational practices.

Empirical evidence regarding acculturation as it relates to the sexual practices and sexual orientation of international student populations provides insight into their assimilation process within the U.S. culture. Such pertinent evidence addresses sexual practice misconceptions and bias when viewing the melding of a culture into its native host's culture. In turn, this will assist in distilling the health, education, and welfare needs of international students as well as natives engaged in cultural assimilation. Just as importantly, such examination will assemble evidence of whether acculturation over time has differing effects on diverse races and ethnicities as well as on males and females. Collectively, such evidence will serve to foster a mutually beneficial commingling of international students within their host nation.

\section{Hypotheses}

The following null hypotheses were tested:

$\mathrm{H}_{1}$ : There are no significant differences between native males and male international students' core masturbatory, oral-genital, or coital practices after five years of exposure to U.S. culture.

H2: There are no significant differences between native males and male international students' procreation practices after five years of exposure to U.S. culture.

H3: There are no significant differences between native males and male international students' sexual orientation after five years of exposure to U.S. culture.

H4: There are no significant differences between native females and female international students' core masturbatory, oral-genital, or coital practices after five years of exposure to U.S. culture. 
H5: There are no significant differences between native females and female international students' procreation practices after five years of exposure to U.S. culture.

H6: There are no significant differences between native females and female international students' sexual orientation after five years of exposure to U.S. culture.

\section{RESEARCH METHOD}

The objective of this study was to describe the consequences of acculturative exposure on international students' core sexual practices, procreation, and sexual orientation. This investigation was a retrospective cross-sectional survey utilizing a convenience sample drawn from the general population of undergraduates at a public, northeastern, metropolitan community college. The college's enrollment was 23,938 with a median age of 22.0 years. In total, 1846 instruments were submitted of which 1830 were coded, which represented $7.6 \%$ of the college's enrollment that semester.

\section{Participants}

The study college ranked $26^{\text {th }}$ nationwide (U.S.) in the number of international students enrolled, collectively hailing from 161 different countries (Institute of International Education, 2017). The college offers 39 AA, AAS, and certificate programs. Respondents were asked to indicate their race/ethnicity, country of birth, U.S. citizenship, years living in the U.S., biological parents' race/ethnicity, and biological parents' country of birth. International students had either legal U.S. alien status (i.e. student visa or resident alien) or illegal (undocumented) alien status. University policy does not allow any record or query distinguishing between the two immigration types. Hereafter both status categories are referenced to as 'alien'. All queries offered an open-ended response. Respondents in this study were from the U.S. and 102 countries and U.S. territorial possessions (Table 1).

A variable matrix was calculated to cross-validate each respondent's native-born status, race/ethnicity, U.S. citizenship, and years living in the U.S. Respondents lacking crossvalidated response sets, with missing data for any of the matrix variables, or offered eccentric responses such as 'citizen of the world'--were eliminated from the sample. Two transgender respondents were removed from the analysis. This resulted in 1661 response sets.

Table 1. International students' country of origin.

\begin{tabular}{lc}
\hline Native country & $\boldsymbol{n}$ \\
\hline Albania & 7 \\
Antigua & 1 \\
Argentina & 1 \\
Armenia & 2 \\
Asia & 1 \\
Azerbaijan & 3 \\
Bangladesh & 17 \\
\end{tabular}




\begin{tabular}{|c|c|}
\hline Native country & $n$ \\
\hline Barbados & 3 \\
\hline Belarus & 2 \\
\hline Belize & 1 \\
\hline Brazil & 5 \\
\hline Burkina Faso & 5 \\
\hline Burma & 2 \\
\hline Cambodia & 1 \\
\hline Cameroon & 2 \\
\hline Canada & 4 \\
\hline Caribbean & 1 \\
\hline Chad & 1 \\
\hline China & 74 \\
\hline Colombia & 10 \\
\hline Costa Rica & 1 \\
\hline Democratic Republic of Congo & 3 \\
\hline Dominica & 1 \\
\hline Dominican Republic & 107 \\
\hline Ecuador & 18 \\
\hline Egypt & 3 \\
\hline England & 2 \\
\hline Finland & 1 \\
\hline France & 3 \\
\hline Gabon & 1 \\
\hline Georgia & 4 \\
\hline Germany & 2 \\
\hline Ghana & 13 \\
\hline Greece & 1 \\
\hline Grenada & 6 \\
\hline Guyana & 1 \\
\hline Guatemala & 3 \\
\hline Guinea & 6 \\
\hline Guyana & 33 \\
\hline Haiti & 11 \\
\hline Honduras & 4 \\
\hline Hong Kong & 3 \\
\hline India & 6 \\
\hline
\end{tabular}




\begin{tabular}{|c|c|}
\hline Native country & $n$ \\
\hline Indonesia & 1 \\
\hline Iraq & 1 \\
\hline Israel & 3 \\
\hline Italy & 2 \\
\hline Ivory Coast & 2 \\
\hline Jamaica & 29 \\
\hline Japan & 2 \\
\hline Jordan & 1 \\
\hline Kazakhstan & 1 \\
\hline Kenya & 1 \\
\hline Korea & 7 \\
\hline Kosovo & 3 \\
\hline Kyrgyzstan & 1 \\
\hline Latvia & 1 \\
\hline Lebanon & 1 \\
\hline Liberia & 1 \\
\hline Malaysia & 1 \\
\hline Mali & 3 \\
\hline Mexico & 12 \\
\hline Mongolia & 1 \\
\hline Montserrat & 1 \\
\hline Montenegro & 2 \\
\hline Morocco & 2 \\
\hline Nassau & 1 \\
\hline Nepal & 4 \\
\hline Nicaragua & 1 \\
\hline Nigeria & 6 \\
\hline Pakistan & 9 \\
\hline Panama & 3 \\
\hline Paraguay & 1 \\
\hline Peru & 2 \\
\hline Philippines & 3 \\
\hline Poland & 9 \\
\hline Puerto Rico & 13 \\
\hline Romania & 1 \\
\hline Russia & 11 \\
\hline
\end{tabular}




\begin{tabular}{lc}
\hline Native country & $\boldsymbol{n}$ \\
\hline Saint Lucia & 9 \\
Saint Vincent & 4 \\
Santo Domingo & 1 \\
Senegal & 6 \\
Serbia & 1 \\
Sierra Leone & 2 \\
South Korea & 6 \\
Sri Lanka & 1 \\
Sweden & 1 \\
Switzerland & 2 \\
Tajikistan & 1 \\
Togo & 2 \\
Trinidad & 1 \\
Tobago & 13 \\
Turkey & 1 \\
U.S. Virgin Islands & 2 \\
Ukraine & 13 \\
United Kingdom & 1 \\
Uzbekistan & 1 \\
Venezuela & 602 \\
Vietnam & 6 \\
Yemen & 6 \\
\hline Total & 1 \\
\hline
\end{tabular}

\section{Procedures}

Respondents were recruited from intact Health Education course sections that were required or elective courses for all but five of the college's degree programs. Respondents were 18 years or older. Consent forms were obtained from each respondent. There were no identifiers linking respondents to their responses. Classroom seating was arranged in formal test-taking configuration. The in-class survey was voluntary, anonymous, and averaged 39 minutes. Respondents opting out of the survey completed an in-class worksheet. Participants placed their instrument or worksheet in a sealed envelope and then into a cloaked ballot box. The University's Institutional Research Review Committee sanctioned the study.

\section{Acculturative Exposure}

Respondents were blocked into the independent variable of 'Acculturative Exposure' (hereafter Acculturative-Exposure) in one of three levels: (1) native born undergraduate and lifelong resident of the U.S. (hereafter Native); (2) international student with alien status and 
U.S. residency of five years or less (hereafter Alien $\leq 5$ year); and (3) international student with alien status and U.S. residency of more than five years (hereafter Alien $>5$ year).

\section{Measures}

The Belcastro Sexual Behavior Inventory (hereafter BSBI-III) gathers in part, comprehensive demographics of respondents' race, ethnicity, country of birth, natal gender, and sexual orientation. The BSBI-III tallies respondents' masturbatory, oral-genital, and coital practices including initiation age, partner's age at initiation, and frequency. Previous studies reported a .85 reliability coefficient for the instrument (Belcastro, 1983; Belcastro, 1985). The instrument's reliability coefficient (Chronbach's Alpha) for the masturbatory, oral-genital, and coital practices under investigation in this study was .91 .

\section{Tests of Significance}

Tests of significance (SPSS IBM Advanced Statistics Version 24.0.0) employed were chisquare $\left(X^{2}\right)$ for nominal variables, one-way ANOVA $(F)$ for scaled variables, Kaplan-Meier life survival analysis, and a stepwise discriminate analysis. Type 1 error rate was set to .05 . For multiple comparisons in the one-way ANOVA, Bonferroni's correction was applied to the Type 1 error rate. Results with insufficient tallies were not reported and results yielding statistically insignificant results were generally not reported.

\section{RESULTS}

Within Acculturative-Exposure 63.8\% $(n=1059)$ were Native, 14.6\% $(n=242)$ Alien $\leq 5$ year, and $21.7 \%(\mathrm{n}=360)$ Alien $>5$ year (Table 2). Regarding race/ethnicity (hereafter race) $10.9 \%(\mathrm{n}$ $=173)$ were White/non-Hispanic (hereafter White), 24.1\% $(\mathrm{n}=382)$ African, $44.6 \%(\mathrm{n}=706)$ Hispanic/Latino, $12.1 \%(\mathrm{n}=192)$ Asian/Pacific Islanders, and 8.2\% $(\mathrm{n}=130)$ Multi-racial. Females outnumbered males in all levels of the independent variable with the exceptions of Multi-racial (Alien $\leq 5$ year) and Asian/Pacific Islanders (Native) (Table 2). Mean age for female respondents was 21.75 years $(n=998)$ and male respondents 21.65 years $(n=652)$. There were no significant differences between this sample's demographics and that of the college's sum enrollment population.

Table 2. Acculturative-exposure by gender and race.

\begin{tabular}{ccccccc}
\hline $\begin{array}{l}\text { Acculturative- } \\
\text { exposure }\end{array}$ & $\begin{array}{c}\text { Total } \\
\mathbf{\%}(\boldsymbol{N})\end{array}$ & $\begin{array}{c}\text { White } \\
\mathbf{\%}(\boldsymbol{n})\end{array}$ & $\begin{array}{c}\text { African } \\
\mathbf{\%}(\boldsymbol{n})\end{array}$ & $\begin{array}{c}\text { Hispanic/ } \\
\text { Latino } \\
\mathbf{\%}(\boldsymbol{n})\end{array}$ & $\begin{array}{c}\text { Asian/ } \\
\text { Pacific } \\
\text { Islander } \\
\mathbf{\%}(\boldsymbol{n})\end{array}$ & $\begin{array}{c}\text { Multi- } \\
\text { racial } \\
\mathbf{\%}(\boldsymbol{n})\end{array}$ \\
\hline Native & $\mathbf{6 3 . 8}$ & $53.8(93)$ & $63.4(242)$ & $75.9(536)$ & $24.0(46)$ & $78.5(102)$ \\
& $(1,059)$ & & & & & \\
Males & $40.8(431)$ & $48.4(45)$ & $40.7(98)$ & $39.3(210)$ & $50.0(23)$ & $36.6(37)$ \\
Females & $59.2(625)$ & $51.6(48)$ & $59.3(143)$ & $60.7(325)$ & $50.0(23)$ & $63.4(64)$ \\
Alien $\leq 5$ yr & $\mathbf{1 4 . 6 ( 2 4 2 )}$ & $20.8(36)$ & $14.4(55)$ & $5.4(38)$ & $49.0(94)$ & $6.2(8)$ \\
$\quad$ Males & $37.2(89)$ & $19.4(7)$ & $45.3(24)$ & $44.7(17)$ & $33.3(31)$ & $50.0(4)$ \\
Females & $62.8(150)$ & $80.6(29)$ & $54.7(29)$ & $55.3(21)$ & $66.7(62)$ & $50.0(4)$ \\
\hline
\end{tabular}




\begin{tabular}{lcccccc}
\hline $\begin{array}{l}\text { Acculturative- } \\
\text { exposure }\end{array}$ & $\begin{array}{c}\text { Total } \\
\%(\boldsymbol{N})\end{array}$ & $\begin{array}{c}\text { White } \\
\mathbf{\%}(\boldsymbol{n})\end{array}$ & $\begin{array}{c}\text { African } \\
\mathbf{\%}(\boldsymbol{n})\end{array}$ & $\begin{array}{c}\text { Hispanic/ } \\
\text { Latino } \\
\mathbf{\%}(\boldsymbol{n})\end{array}$ & $\begin{array}{c}\text { Asian/ } \\
\text { Pacific } \\
\text { Islander } \\
\mathbf{\%}(\boldsymbol{n})\end{array}$ & $\begin{array}{c}\text { Multi- } \\
\text { racial } \\
\%(\boldsymbol{n})\end{array}$ \\
\hline Alien $>5 \mathrm{yr}$ & $\mathbf{2 1 . 7 ( 3 6 0 )}$ & $25.4(44)$ & $22.3(85)$ & $18.7(132)$ & $27.1(52)$ & $15.4(20)$ \\
Males & $37.2(132)$ & $29.5(13)$ & $32.9(27)$ & $42.0(55)$ & $42.3(22)$ & $31.6(6)$ \\
Females & $62.8(223)$ & $70.5(31)$ & $67.1(55)$ & $58.0(76)$ & $57.7(30)$ & $68.4(13)$ \\
\hline
\end{tabular}

Native respondents were significantly younger $(\bar{X}=20.96$ years $)$ than Alien $\leq 5$ year $(\bar{X}=$ 22.67 years $)$ and Alien $>5$ year $(\bar{X}=23.36$ years $)$ respondents, with a relatively diminutive effect size. There were significant differences in Acculturative-Exposure and the number of biological and non-biological siblings. Native had significantly less biological brothers and sisters than Alien $>5$ year, with a diminutive effect size. Native had significantly more brothers and sisters from different parents than Alien $\leq 5$ year, with a diminutive effect size. African Native had significantly less biological brothers from the same parents than Alien $>5$ year, with a diminutive effect size. African Alien $\leq 5$ year had significantly less biological brothers from the same parents than Alien $>5$ year, with a diminutive effect size (Table 3 ).

Table 3. Acculturative-exposure demographics.

\begin{tabular}{lccccccc}
\hline Demographic & $\boldsymbol{n}$ & $\boldsymbol{M}$ & $\boldsymbol{S D}$ & $\boldsymbol{F}$ & $\mathbf{d f 1 / \mathbf { d f 2 }}$ & $\begin{array}{c}\boldsymbol{p} \\
\text { value }\end{array}$ & $\boldsymbol{\eta}^{\mathbf{2}}$ \\
\hline Age & & & & 34.395 & 2,1571 & .000 & .04 \\
$\quad$ Native & 1,018 & 20.962 & 4.354 & & & & \\
$\quad$ Alien $\leq 5 \mathrm{yr}$ & 226 & $22.672^{*}$ & 5.476 & & & & \\
Alien $>5 \mathrm{yr}$ & 330 & $23.363^{*}$ & 6.124 & & &
\end{tabular}

Biological siblings

Brothers-same

biological parents

$\begin{array}{llll}5.660 & 2,1385 \quad .020 \dagger & .008\end{array}$

$\begin{array}{lccc}\text { Native } & 888 & 0.965 & 1.082 \\ \text { Alien } \leq 5 \mathrm{yr} & 200 & 1.085 & 1.097 \\ \text { Alien }>5 \mathrm{yr} & 300 & 1.233^{*} & 1.585\end{array}$

Sisters-same

biological parents

$\begin{array}{lccc}\text { Native } & 866 & 0.919 & 1.082 \\ \text { Alien } \leq 5 \mathrm{yr} & 203 & 1.004 & 1.031 \\ \text { Alien }>5 \mathrm{yr} & 299 & 1.307^{*} & 1.714\end{array}$

Brothers-different

biological parents

$\begin{array}{llll}6.739 & 2,1204 \quad .000 \dagger & .01\end{array}$

Native

$806 \quad 1.060 \quad 1.672$




\begin{tabular}{|c|c|c|c|c|c|c|c|}
\hline Demographic & $n$ & $M$ & $S D$ & $F$ & df1/ df2 & $\begin{array}{c}p \\
\text { value }\end{array}$ & $\eta^{2}$ \\
\hline Alien $\leq 5 \mathrm{yr}$ & 156 & $0.538 *$ & 1.321 & & & & \\
\hline Alien $>5 \mathrm{yr}$ & 245 & 0.983 & 1.649 & & & & \\
\hline $\begin{array}{l}\text { Sisters-different } \\
\text { biological parents }\end{array}$ & & & & 7.591 & 2,1200 & $.000^{\dagger}$ & .01 \\
\hline Native & 796 & 1.133 & 1.772 & & & & \\
\hline Alien $\leq 5 \mathrm{yr}$ & 156 & $0.570 *$ & 1.060 & & & & \\
\hline Alien $>5 \mathrm{yr}$ & 251 & 0.976 & 1.622 & & & & \\
\hline \multicolumn{8}{|l|}{ African } \\
\hline $\begin{array}{l}\text { Brothers-same } \\
\text { biological parents }\end{array}$ & & & & 5.429 & 2,307 & $.048^{\dagger}$ & .03 \\
\hline Native & 193 & 1.031 & 1.215 & & & & \\
\hline Alien $\leq 5$ yr & 49 & 1.591 & 1.470 & & & & \\
\hline Alien $>5 \mathrm{yr}$ & 68 & $1.720 *$ & 2.613 & & & & \\
\hline $\begin{array}{l}\text { Brothers-different } \\
\text { biological parents }\end{array}$ & & & & 4.114 & 2,278 & $.041^{\dagger}$ & .03 \\
\hline Native & 184 & 1.440 & 1.707 & & & & \\
\hline Alien $\leq 5 \mathrm{yr}$ & 42 & $0.857 * *$ & 1.260 & & & & \\
\hline Alien $>5 \mathrm{yr}$ & 55 & $1.890 * *$ & 2.199 & & & & \\
\hline
\end{tabular}

* Significant difference versus Native

** Significant difference

$\dagger$ Tamhane's post hoc

There was a significant difference in sexual orientation between the three levels of Acculturative-Exposure. While $15.4 \%$ of Native were homosexual or bisexual only $8.1 \%$ of Alien $\leq 5$ year were homosexual or bisexual-with Alien $>5$ year in the middle at $10.3 \%$. There were no significant differences in sexual orientation between the three levels of AcculturativeExposure for males. There were significant differences in sexual orientation between the three levels of Acculturative-Exposure for females. While $19.1 \%$ of Native females were homosexual or bisexual only $6.5 \%$ of Alien $\leq 5$ year females were homosexual or bisexualwith Alien $>5$ year females in the middle at $10.5 \%$ (Table 4 ).

Table 4. Acculturative-exposure and sexual orientation.

\begin{tabular}{lccc}
\hline & $\begin{array}{c}\text { Total }^{\mathbf{a}} \\
\mathbf{\%}(\boldsymbol{n})\end{array}$ & $\begin{array}{c}\text { Males } \\
\mathbf{\%}(\boldsymbol{n})\end{array}$ & $\begin{array}{c}\text { Females }^{\mathbf{b}} \\
\mathbf{\%}(\boldsymbol{n})\end{array}$ \\
\hline $\begin{array}{l}\text { Native } \\
\text { Homosexual }\end{array}$ & $6.9(70)$ & $7.3(30)$ & $6.7(40)$ \\
Bisexual & $8.5(86)$ & $2.7(11)$ & $12.4(74)$ \\
\hline
\end{tabular}




\begin{tabular}{lccc}
\hline & $\begin{array}{c}\text { Total }^{\mathbf{a}} \\
\mathbf{\%}(\boldsymbol{n})\end{array}$ & $\begin{array}{c}\text { Males } \\
\mathbf{\%}(\boldsymbol{n})\end{array}$ & $\begin{array}{c}\text { Females }^{\mathbf{b}} \\
\mathbf{\%}(\boldsymbol{n})\end{array}$ \\
\hline Heterosexual & $84.6(855)$ & $90.0(371)$ & $80.9(482)$ \\
Alien $\leq 5$ yr & & & \\
$\quad$ Homosexual & $5.4(12)$ & $8.5(7)$ & $3.6(5)$ \\
Bisexual & $2.7(6)$ & $2.4(2)$ & $2.9(4)$ \\
$\quad$ Heterosexual & $91.9(205)$ & $89.0(73)$ & $93.5(130)$ \\
Alien $>5$ yr & & & \\
Homosexual & $4.7(16)$ & $6.3(8)$ & $3.8(8)$ \\
Bisexual & $5.6(19)$ & $3.2(4)$ & $6.7(14)$ \\
$\quad$ Heterosexual & $89.6(303)$ & $90.5(114)$ & $89.4(186)$ \\
\hline $\mathrm{a}\left(X^{2}(4, \mathrm{~N}=1572)=13.838, \mathrm{p}=0.008\right)$ & & \\
$\mathrm{b}\left(X^{2}(4, \mathrm{~N}=943)=19.313, \mathrm{p}=0.001\right)$ & &
\end{tabular}

\section{Marriage}

There were significant differences in Acculturative-Exposure marital proportions for males and females. While $4.6 \%(n=18)$ of Native males were married, 7.3\% $(n=6)$ of male Alien $\leq 5$ year and $10.7 \%(\mathrm{n}=13)$ of male Alien $>5$ year were married $\left(X^{2}(2, \mathrm{~N}=595)=6.197, \mathrm{p}=\right.$ $0.045)$. While $6.1 \%(n=31)$ of Native females were married, $16.0 \%(n=19)$ of female Alien $\leq 5$ year and $19.9 \%(\mathrm{n}=35)$ of female Alien $>5$ year were married $\left(X^{2}(2, \mathrm{~N}=806)=30.851, \mathrm{p}=\right.$ $0.000)$.

\section{Procreation and Abortion}

There were no significant differences in fecundity, pregnancy outcome, pregnancy marital status, or abortion experience between Native males and male international students in U.S. residency for five years or less or more than five years.

Native females were significantly younger than Alien $>5$ year females at first pregnancy with a robust effect size. Native females partners were significantly younger than Alien $\leq 5$ year and Alien $>5$ year females at first pregnancy with a robust effect size (Table 5). Significantly more Alien $>5$ year females $(36.7 \%)$ than Native $(29.9 \%)$ and Alien $\leq 5$ year $(14.0 \%)$ females had ever been pregnant (Table 6).

Significantly more Alien $>5$ year $(28.1 \%)$ females than Native $(5.3 \%)$ or Alien $\leq 5$ year females $(26.7 \%)$ were married to the father of their first pregnancy. Significantly more Native females $(45.0 \%)$ than Alien $>5$ year $(37.5 \%)$ or Alien $\leq 5$ year females $(33.3 \%)$ were cohabiting with the father of their first pregnancy. Significantly more Native females $(49.7 \%)$ than Alien $>5$ year $(34.4 \%)$ or Alien $\leq 5$ year females $(40.0 \%)$ were single for their first pregnancy (Table 6).

Significantly more first pregnancies of Native females (14.1\%) and Alien $>5$ year females (13.3\%) resulted in a miscarriage than Alien $\leq 5$ year females (6.7\%). Significantly more first pregnancies of Alien $>5$ year females $(58.3 \%)$ than Native $(27.6 \%)$ or Alien $\leq 5$ year females $(26.7 \%)$ resulted in a live birth. Significantly more first pregnancies of Alien $\leq 5$ year females (66.7\%) and Native (58.3\%) females than Alien>5 year female (28.3\%) resulted in an abortion. 
Significantly more Alien $\leq 5$ year females $(66.7 \%)$ than Native $(43.5 \%)$ or Alien $>5$ year females $(27.9 \%)$ wanted an abortion in their first pregnancy. Significantly more conceptus fathers of Alien $\leq 5$ year females $(73.3 \%)$ than Native $(33.3 \%)$ or Alien $>5$ year females $(26.2 \%)$ wanted an abortion in their first pregnancy (Table 6).

Native females were significantly younger than Alien $\leq 5$ year and Alien $>5$ year females at second pregnancy with a robust effect size. Male partners of Native females were significantly younger at females' second pregnancy than Alien $>5$ year females' partners with a robust effect size (Table 5).

Table 5. Acculturative-exposure and fecundity.

\begin{tabular}{|c|c|c|c|c|c|c|c|}
\hline & $n$ & $M$ & $S D$ & $\boldsymbol{F}$ & df1/df2 & $p$ value & $\eta^{2}$ \\
\hline $\begin{array}{l}\text { Females' age at first } \\
\text { pregnancy }\end{array}$ & & & & 18.798 & 2,235 & $.000^{\dagger}$ & .14 \\
\hline Native & 159 & 18.150 & 2.929 & & & & \\
\hline Alien $\leq 5 \mathrm{yr}$ & 16 & 19.875 & 3.242 & & & & \\
\hline Alien $>5 \mathrm{yr}$ & 63 & $21.254^{*}$ & 4.522 & & & & \\
\hline $\begin{array}{l}\text { Partner's age at } \\
\text { female's first } \\
\text { pregnancy }\end{array}$ & & & & 30.844 & 2,231 & $.000^{\dagger}$ & .21 \\
\hline Native & 157 & 20.331 & 3.903 & & & & \\
\hline Alien $\leq 5 \mathrm{yr}$ & 16 & $24.125^{*}$ & 4.745 & & & & \\
\hline Alien $>5 \mathrm{yr}$ & 61 & $25.426^{*}$ & 5.581 & & & & \\
\hline $\begin{array}{l}\text { Females' age at } \\
\text { second pregnancy }\end{array}$ & & & & 7.332 & 2,95 & .001 & .13 \\
\hline Native & 64 & 20.187 & 3.468 & & & & \\
\hline Alien $\leq 5 \mathrm{yr}$ & 9 & $23.888 *$ & 6.112 & & & & \\
\hline Alien $>5 \mathrm{yr}$ & 25 & $23.360 *$ & 4.715 & & & & \\
\hline $\begin{array}{l}\text { Partner's age at } \\
\text { female's second } \\
\text { pregnancy }\end{array}$ & & & & 9.128 & 2,90 & .000 & .17 \\
\hline Native & 62 & 23.629 & 5.393 & & & & \\
\hline Alien $\leq 5$ yr & 9 & 31.444 & 11.990 & & & & \\
\hline Alien $>5 \mathrm{yr}$ & 22 & $28.454^{*}$ & 5.603 & & & & \\
\hline
\end{tabular}

\footnotetext{
* Significant difference versus Native

$\dagger$ Tamhane's post hoc
} 
Table 6. Acculturative-exposure and first pregnancy outcome.

\begin{tabular}{|c|c|c|c|c|c|c|c|c|c|c|}
\hline & \multicolumn{5}{|c|}{ Females } & \multicolumn{5}{|c|}{ Males } \\
\hline & $\%$ & $n$ & $X^{2}$ & $d f$ & $\underset{\text { value }}{p}$ & $\%$ & $n$ & $X^{2}$ & $d f$ & $\underset{\text { value }}{p}$ \\
\hline $\begin{array}{l}\text { Ever pregnant/ } \\
\text { impregnate }\end{array}$ & & 842 & 18.468 & 2 & .000 & & 519 & & & \\
\hline Native & 29.9 & & & & & 19.0 & & & & \\
\hline Alien $\leq 5 \mathrm{yr}$ & 14.0 & & & & & 25.4 & & & & \\
\hline Alien $>5 \mathrm{yr}$ & 36.7 & & & & & 28.1 & & & & \\
\hline Outcome of pregnancy & & 231 & 20.871 & 2 & .000 & & 101 & & & \\
\hline \multicolumn{11}{|l|}{ Miscarriage } \\
\hline Native & 14.1 & & & & & 25.4 & & & & \\
\hline Alien $\leq 5 \mathrm{yr}$ & 6.7 & & & & & 7.7 & & & & \\
\hline Alien $>5 \mathrm{yr}$ & 13.3 & & & & & 24.0 & & & & \\
\hline \multicolumn{11}{|l|}{ Abortion } \\
\hline Native & 58.3 & & & & & 54.0 & & & & \\
\hline Alien $\leq 5 \mathrm{yr}$ & 66.7 & & & & & 69.2 & & & & \\
\hline Alien $>5 \mathrm{yr}$ & 28.3 & & & & & 52.0 & & & & \\
\hline \multicolumn{11}{|l|}{ Birth } \\
\hline Native & 27.6 & & & & & 20.6 & & & & \\
\hline Alien $\leq 5 \mathrm{yr}$ & 26.7 & & & & & 23.1 & & & & \\
\hline Alien $>5 \mathrm{yr}$ & 58.3 & & & & & 24.0 & & & & \\
\hline $\begin{array}{l}\text { Relationship in } \\
\text { pregnancy }\end{array}$ & & 230 & 23.572 & 2 & .000 & & 100 & & & \\
\hline \multicolumn{11}{|l|}{ Married } \\
\hline Native & 5.3 & & & & & 6.6 & & & & \\
\hline Alien $\leq 5 \mathrm{yr}$ & 26.7 & & & & & 12.5 & & & & \\
\hline Alien $>5 \mathrm{yr}$ & 28.1 & & & & & 13.0 & & & & \\
\hline \multicolumn{11}{|l|}{ Cohabiting } \\
\hline Native & 45.0 & & & & & 31.1 & & & & \\
\hline Alien $\leq 5 \mathrm{yr}$ & 33.3 & & & & & 12.5 & & & & \\
\hline Alien $>5 \mathrm{yr}$ & 37.5 & & & & & 39.2 & & & & \\
\hline \multicolumn{11}{|l|}{ Single } \\
\hline Native & 49.7 & & & & & 62.3 & & & & \\
\hline Alien $\leq 5 \mathrm{yr}$ & 40.0 & & & & & 75.0 & & & & \\
\hline Alien $>5 \mathrm{yr}$ & 34.4 & & & & & 47.8 & & & & \\
\hline
\end{tabular}




\begin{tabular}{|c|c|c|c|c|c|c|c|c|c|}
\hline & \multicolumn{5}{|c|}{ Females } & \multicolumn{4}{|c|}{ Males } \\
\hline & $\%$ & $n$ & $X^{2}$ & $d f$ & $\begin{array}{c}p \\
\text { value }\end{array}$ & $\%$ & $n$ & $X^{2} \quad d f$ & $\underset{\text { value }}{p}$ \\
\hline $\begin{array}{l}\text { You wanted to abort } \\
\text { pregnancy }\end{array}$ & & 230 & 8.840 & 2 & .012 & & 101 & & \\
\hline Native & 43.5 & & & & & 59.7 & & & \\
\hline Alien $\leq 5 \mathrm{yr}$ & 66.7 & & & & & 41.2 & & & \\
\hline Alien $>5 \mathrm{yr}$ & 27.9 & & & & & 50.0 & & & \\
\hline $\begin{array}{l}\text { Partner wanted to abort } \\
\text { pregnancy }\end{array}$ & & 227 & 11.913 & 2 & .003 & & 100 & & \\
\hline Native & 33.3 & & & & & 40.3 & & & \\
\hline Alien $\leq 5 \mathrm{yr}$ & 73.3 & & & & & 43.8 & & & \\
\hline Alien $>5 \mathrm{yr}$ & 26.2 & & & & & 50.0 & & & \\
\hline
\end{tabular}

\section{Initiation Age}

Respondents reported their initiation age for a compendium of coital events. Native males were significantly younger $(\overline{\mathrm{X}}=15.40$ years $)$ than Alien $\leq 5$ year $(\overline{\mathrm{X}}=17.23$ years $)$ males at their first coitus, with a relatively small effect size. Native male coital partners were significantly younger $(\bar{X}=16.34$ years) than Alien $\leq 5$ year $(\bar{X}=18.23$ years $)$ male coital partners at the male's first coitus, with a relatively small effect size (Table 7.1). There was no significant difference between Native, Alien $\leq 5$ year, and Alien $>5$ year males and their number of lifetime coital partners. Native males were significantly younger $(\overline{\mathrm{X}}=17.05$ year $)$ than Alien $\leq 5$ year $(\overline{\mathrm{X}}$ $=20.20$ years) males at first interracial coitus, with a relatively small effect size (Table 7.1$)$.

Table 7.1. Male coital initiation ages.

\begin{tabular}{|c|c|c|c|c|c|c|c|}
\hline & $n$ & $M$ & $S D$ & $F$ & df1/df2 & $p$ value & $\eta^{2}$ \\
\hline Age at first coitus & & & & 11.438 & 2,456 & $.002 \dagger$ & .04 \\
\hline Native & 315 & 15.403 & 2.241 & & & & \\
\hline Alien $\leq 5 \mathrm{yr}$ & 55 & $17.234^{*}$ & 3.706 & & & & \\
\hline Alien $>5 \mathrm{yr}$ & 89 & 15.684 & 3.047 & & & & \\
\hline $\begin{array}{l}\text { Partner's age at male's } \\
\text { first coitus }\end{array}$ & & & & 5.861 & 2,439 & $.023^{\dagger}$ & .05 \\
\hline Native & 303 & 16.343 & 3.464 & & & & \\
\hline Alien $\leq 5 \mathrm{yr}$ & 51 & $18.235^{*}$ & 4.688 & & & & \\
\hline Alien $>5 \mathrm{yr}$ & 88 & 16.750 & 3.677 & & & & \\
\hline $\begin{array}{l}\text { Age at first interracial } \\
\text { coitus }\end{array}$ & & & & 6.828 & 2,237 & .001 & .05 \\
\hline Native & 193 & 17.051 & 2.641 & & & & \\
\hline Alien $\leq 5 \mathrm{yr}$ & 10 & $20.200 *$ & 4.541 & & & & \\
\hline
\end{tabular}




\begin{tabular}{cccccccc}
\hline & $\boldsymbol{n}$ & $\boldsymbol{M}$ & $\boldsymbol{S D}$ & $\boldsymbol{F}$ & df1/df2 & $\boldsymbol{p}$ value & $\boldsymbol{\eta}^{\mathbf{2}}$ \\
\hline Alien $>5 \mathrm{yr}$ & 37 & 17.973 & 3.451 & & & & \\
\hline
\end{tabular}

* Significant difference versus Native

$\dagger$ Tamhane's post hoc

Native females were significantly younger $(\overline{\mathrm{X}}=15.81$ years $)$ than Alien $>5$ year $(\overline{\mathrm{X}}=16.82$ years) and Alien $\leq 5$ year $(\overline{\mathrm{X}}=18.15$ year) females at first coitus, with a relatively large effect size. Native females' coital partners were significantly younger $(\overline{\mathrm{X}}=17.67$ years $)$ than Alien $>5$ year $(\overline{\mathrm{X}}=19.74$ years $)$ and Alien $\leq 5$ year $(\overline{\mathrm{X}}=22.37$ years $)$ females' coital partners at the female's first coitus with a large effect size (Table 7.2).There was no significant difference between Native, Alien $\leq 5$ year, and Alien $>5$ year females and their number of lifetime coital partners.

Native females' coital partners were significantly younger $(\overline{\mathrm{X}}=19.32$ years $)$ than Alien $\leq 5$ year $(\overline{\mathrm{X}}=26.70$ years) females' coital partners at first interracial coitus, with a relatively moderate effect size (Table 7.2).Native females were significantly younger $(\overline{\mathrm{X}}=17.69$ years) than Alien $\leq 5$ year ( $\bar{X}=20.45$ year) females at first coitus while using marijuana, with a relatively large effect size. Native females' coital partners were significantly younger $(\overline{\mathrm{X}}=$ 19.65 year) than Alien $>5$ year $(\bar{X}=23.00$ years $)$ and Alien $\leq 5$ year $(\bar{X}=25.36$ years $)$ females' coital partners at first coitus while using marijuana, with a large effect size (Table 7.2).

Table 7.2. Female coital initiation ages.

\begin{tabular}{|c|c|c|c|c|c|c|c|}
\hline & $n$ & $M$ & $S D$ & $\boldsymbol{F}$ & df1/df2 & $p$ value & $\eta^{2}$ \\
\hline Age at first coitus & & & & 41.114 & 2,715 & $.000 \dagger$ & .10 \\
\hline Native & 474 & 15.816 & 1.957 & & & & \\
\hline Alien $\leq 5 \mathrm{yr}$ & 82 & $18.158 *$ & 3.144 & & & & \\
\hline Alien $>5 \mathrm{yr}$ & 162 & $16.827^{*}$ & 2.733 & & & & \\
\hline $\begin{array}{l}\text { Partner's age at } \\
\text { female's first coitus }\end{array}$ & & & & 70.140 & 2,686 & $.000^{\dagger}$ & .17 \\
\hline Native & 461 & 17.674 & 2.819 & & & & \\
\hline Alien $\leq 5 \mathrm{yr}$ & 79 & $22.379 *$ & 5.400 & & & & \\
\hline Alien $>5 \mathrm{yr}$ & 149 & $19.745^{*}$ & 4.077 & & & & \\
\hline $\begin{array}{l}\text { Partner's age at } \\
\text { female's first interracial } \\
\text { coitus }\end{array}$ & & & & 27.407 & 2,252 & .001 & .05 \\
\hline Native & 189 & 19.322 & 4.153 & & & & \\
\hline Alien $\leq 5 \mathrm{yr}$ & 24 & $26.708^{*}$ & 7.898 & & & & \\
\hline Alien $>5 \mathrm{yr}$ & 42 & 21.785 & 5.158 & & & & \\
\hline $\begin{array}{l}\text { Age at first coitus with } \\
\text { marijuana }\end{array}$ & & & & 10.504 & 2,209 & $.026^{\dagger}$ & .09 \\
\hline
\end{tabular}




\begin{tabular}{lrcccccc}
\hline & \multicolumn{1}{c}{$\boldsymbol{n}$} & $\boldsymbol{M}$ & $\boldsymbol{S D}$ & $\boldsymbol{F}$ & $\mathbf{d f 1 / d f 2}$ & $\boldsymbol{p}$ value & $\boldsymbol{\eta}^{\mathbf{2}}$ \\
\hline Native & 176 & 17.698 & 2.350 & & & & \\
Alien $\leq 5 \mathrm{yr}$ & 11 & $20.454^{*}$ & 2.805 & & & & \\
$\quad$ Alien >5 yr & 25 & 19.520 & 3.765 & & & & \\
Partner's age at & & & & 16.382 & 2,205 & $.014^{\dagger}$ & .14 \\
$\begin{array}{l}\text { female's first coitus } \\
\text { with marijuana }\end{array}$ & & & & & & & \\
$\quad$ Native & 172 & 19.657 & 3.644 & & & & \\
Alien $\leq 5 \mathrm{yr}$ & 11 & $25.363^{*}$ & 5.258 & & & & \\
Alien $>5$ yr & 25 & $23.000^{*}$ & 5.766 & & & & \\
\hline
\end{tabular}

* Significant difference versus native

$\dagger$ Tamhane's post hoc

\section{Lifetime Oral-Genital, Coital, and Anal Penetration Experience}

Respondents reported whether they ever experienced a compendium of oral-genital, coital, and anal penetration events in their lifetime. Significantly more Native males (74.9\%) than Alien $>5$ year $(71.2 \%)$ and Alien $\leq 5$ year $(60.7 \%)$ males ever received female performed fellatio. Significantly more Native males (62.4\%) than Alien $>5$ year $(56.1 \%)$ and Alien $\leq 5$ year $(40.4 \%)$ males ever performed cunnilingus. Significantly more Native males (48.3\%) than Alien $>5$ year $(38.6 \%)$ and Alien $\leq 5$ year $(24.7 \%)$ males ever performed cunnilingus while receiving fellatio from a female. Significantly more Native males $(14.6 \%)$ than Alien $>5$ year $(9.1 \%)$ and Alien $\leq 5$ year (5.6\%) males ever performed analingus on a female (Table 8.1).

Significantly more Native males $(33.9 \%)$ than Alien $>5$ year $(28.8 \%)$ and Alien $\leq 5$ year (22.5\%) males ever had coitus with a female they knew for less than 24 hours. Significantly more Native males (13.9\%) than Alien $>5$ year $(12.1 \%)$ and Alien $\leq 5$ year $(3.4 \%)$ males ever had coitus with a female they met on the internet. Significantly more Native males (11.4\%) than Alien $>5$ year $(9.8 \%)$ and Alien $\leq 5$ year $(2.2 \%)$ males ever had simultaneous coitus with two females. Significantly more Native males $(44.8 \%)$ than Alien $>5$ year $(28.0 \%)$ and Alien $\leq 5$ year (11.2\%) males ever had interracial coitus. Significantly more Native males $(40.1 \%)$ than Alien $>5$ year $(29.5 \%)$ and Alien $\leq 5$ year $(20.2 \%)$ males ever had coitus while consuming alcohol. Significantly more Native males (27.8\%) than Alien $>5$ year $(16.7 \%)$ and Alien $\leq 5$ year (3.4\%) males ever had coitus while using marijuana. Significantly more Native males (10.9\%) than Alien $>5$ year $(3.9 \%)$ and Alien $\leq 5$ year $(1.1 \%)$ males ever had coitus while the female was bound. Significantly more Native males (33.9\%) than Alien $>5$ year $(25.0 \%)$ and Alien $\leq 5$ year $(20.2 \%)$ males ever had coitus after spanking the female. Significantly more Native males (27.1\%) than Alien $>5$ year $(15.2 \%)$ and Alien $\leq 5$ year $(11.2 \%)$ males ever had coitus after administering pain to the female. Significantly more Native males $(10.0 \%)$ than Alien $>5$ year $(3.8 \%)$ and Alien $\leq 5$ year $(2.2 \%)$ males ever had coitus after receiving pain from the female. Significantly more Native males (51.0\%) than Alien $>5$ year (40.9\%) and Alien $\leq 5$ year (22.5\%) males ever had a coital orgasm. Significantly more Native males $(24.4 \%)$ than Alien $>5$ year $(19.7 \%)$ and Alien $\leq 5$ year $(7.9 \%)$ males ever photographed or videotaped themselves during coitus. However, there was no significant difference in males' acculturation exposure and their ever hiring a female prostitute (Table 8.1). 
Table 8.1. Male ever-experienced oral-genital and coital practices.

\begin{tabular}{|c|c|c|c|c|c|}
\hline & $\%$ & $n$ & $x^{2}$ & $d f$ & $p$ value \\
\hline Received female performed fellatio & & 652 & 7.575 & 2 & .023 \\
\hline Native & 74.9 & & & & \\
\hline Alien $\leq 5 \mathrm{yr}$ & 60.7 & & & & \\
\hline Alien $>5 \mathrm{yr}$ & 71.2 & & & & \\
\hline Performed cunnilingus & & 652 & 14.911 & 2 & .001 \\
\hline Native & 62.4 & & & & \\
\hline Alien $\leq 5 \mathrm{yr}$ & 40.4 & & & & \\
\hline Alien $>5 \mathrm{yr}$ & 56.1 & & & & \\
\hline $\begin{array}{l}\text { Performed cunnilingus as female } \\
\text { performed fellatio }\end{array}$ & & 652 & 18.013 & 2 & .000 \\
\hline Native & 48.3 & & & & \\
\hline Alien $\leq 5 \mathrm{yr}$ & 24.7 & & & & \\
\hline Alien $>5 \mathrm{yr}$ & 38.6 & & & & \\
\hline Performed analingus on a female & & 652 & 7.104 & 2 & .029 \\
\hline Native & 14.6 & & & & \\
\hline Alien $\leq 5 \mathrm{yr}$ & 5.6 & & & & \\
\hline Alien $>5 \mathrm{yr}$ & 9.1 & & & & \\
\hline Ever had coitus & & 652 & & & \\
\hline Native & 62.7 & & & & \\
\hline Alien $\leq 5 \mathrm{yr}$ & 77.3 & & & & \\
\hline Alien $>5 \mathrm{yr}$ & 66.1 & & & & \\
\hline $\begin{array}{l}\text { Ever had coitus with partner known } \\
\text { for }<24 \text { hours }\end{array}$ & & 652 & 11.529 & 2 & .003 \\
\hline Native & 26.9 & & & & \\
\hline Alien $\leq 5 \mathrm{yr}$ & 18.0 & & & & \\
\hline Alien $>5 \mathrm{yr}$ & 13.6 & & & & \\
\hline $\begin{array}{l}\text { Ever had coitus with female met on } \\
\text { the internet }\end{array}$ & & 652 & 7.711 & 2 & .021 \\
\hline Native & 13.9 & & & & \\
\hline Alien $\leq 5 \mathrm{yr}$ & 3.4 & & & & \\
\hline Alien $>5 \mathrm{yr}$ & 12.1 & & & & \\
\hline $\begin{array}{l}\text { Ever had simultaneous coitus with } \\
\text { two females }\end{array}$ & & 652 & 6.934 & 2 & .031 \\
\hline Native & 11.4 & & & & \\
\hline
\end{tabular}




\begin{tabular}{|c|c|c|c|c|c|}
\hline & $\%$ & $n$ & $X^{2}$ & $d f$ & $p$ value \\
\hline Alien $\leq 5 \mathrm{yr}$ & 2.2 & & & & \\
\hline Alien $>5 \mathrm{yr}$ & 9.8 & & & & \\
\hline Ever had interracial coitus & & 652 & 41.168 & 2 & .000 \\
\hline Native & 44.8 & & & & \\
\hline Alien $\leq 5 \mathrm{yr}$ & 11.2 & & & & \\
\hline Alien $>5 \mathrm{yr}$ & 28.0 & & & & \\
\hline Ever had coitus with alcohol & & 652 & 15.194 & 2 & .001 \\
\hline Native & 40.1 & & & & \\
\hline Alien $\leq 5 \mathrm{yr}$ & 20.2 & & & & \\
\hline Alien $>5 \mathrm{yr}$ & 29.5 & & & & \\
\hline Ever had coitus with marijuana & & 652 & 28.517 & 2 & .000 \\
\hline Native & 27.8 & & & & \\
\hline Alien $\leq 5 \mathrm{yr}$ & 3.4 & & & & \\
\hline Alien $>5 \mathrm{yr}$ & 16.7 & & & & \\
\hline $\begin{array}{l}\text { Ever had coitus while female was } \\
\text { bound }\end{array}$ & & 652 & 13.627 & 2 & .001 \\
\hline Native & 10.9 & & & & \\
\hline Alien $\leq 5 \mathrm{yr}$ & 1.1 & & & & \\
\hline Alien $>5 \mathrm{yr}$ & 3.9 & & & & \\
\hline $\begin{array}{l}\text { Ever had coitus after spanking the } \\
\text { female }\end{array}$ & & 652 & 8.653 & 2 & .013 \\
\hline Native & 33.9 & & & & \\
\hline Alien $\leq 5 \mathrm{yr}$ & 20.2 & & & & \\
\hline Alien $>5 \mathrm{yr}$ & 25.0 & & & & \\
\hline $\begin{array}{l}\text { Ever had coitus after administering } \\
\text { pain to the female }\end{array}$ & & 652 & 15.875 & 2 & .000 \\
\hline Native & 27.1 & & & & \\
\hline Alien $\leq 5 \mathrm{yr}$ & 11.2 & & & & \\
\hline Alien $>5 \mathrm{yr}$ & 15.2 & & & & \\
\hline $\begin{array}{l}\text { Ever had coitus after receiving pain } \\
\text { from the female }\end{array}$ & & 652 & 9.745 & 2 & .008 \\
\hline Native & 10.0 & & & & \\
\hline Alien $\leq 5 \mathrm{yr}$ & 2.2 & & & & \\
\hline Alien $>5 \mathrm{yr}$ & 3.8 & & & & \\
\hline Ever had a coital orgasm & & 652 & 25.493 & 2 & .000 \\
\hline
\end{tabular}




\begin{tabular}{lccccc}
\hline & $\mathbf{\%}$ & $\boldsymbol{n}$ & $\boldsymbol{X}^{\mathbf{2}}$ & $\boldsymbol{d f}$ & $\boldsymbol{p}$ value \\
\hline Native & 51.0 & & & & \\
Alien $\leq 5 \mathrm{yr}$ & 22.5 & & & & \\
$\quad$ Alien $>5 \mathrm{yr}$ & 40.9 & & & & \\
Ever photographed or videotaped & & 652 & 12.245 & 2 & .002 \\
during coitus & & & & & \\
$\quad$ Native & 24.4 & & & & \\
$\quad$ Alien $\leq 5 \mathrm{yr}$ & 7.9 & & & & \\
$\quad$ Alien $>5$ yr & 19.7 & & & & \\
Ever paid for a prostitute & & 652 & 0.480 & 2 & .787 \\
$\quad$ Native & 3.5 & & & & \\
$\quad$ Alien $\leq 5$ yr & 3.4 & & & & \\
$\quad$ Alien $>5$ yr & 2.3 & & & & \\
\hline
\end{tabular}

Significantly more Native females $(18.3 \%)$ than Alien $>5$ year $(9.1 \%)$ and Alien $\leq 5$ year (5.0\%) females ever received female performed cunnilingus. Significantly more Native females $(71.0 \%)$ than Alien $>5$ year $(64.9 \%)$ and Alien $\leq 5$ year $(48.9 \%)$ females ever received male performed cunnilingus. Significantly more Native females (62.1\%) than Alien>5 year (56.2\%) and Alien $\leq 5$ year $(44.6 \%)$ females ever performed fellatio. Significantly more Native females $(17.3 \%)$ than Alien $>5$ year $(10.6 \%)$ and Alien $\leq 5$ year $(6.5 \%)$ females ever performed cunnilingus. Significantly more Native females (47.0\%) than Alien>5 year (42.8\%) and Alien $\leq 5$ year (35.3\%) females ever performed fellatio while receiving cunnilingus from a male. Significantly more Native females (27.2\%) than Alien $>5$ year $(20.2 \%)$ and Alien $\leq 5$ year (12.9\%) females ever received male performed analingus (Table 8.2).

Significantly more Native females $(76.3 \%)$ than Alien $>5$ year $(73.6 \%)$ and Alien $\leq 5$ year (56.8\%) females ever had coitus. Significantly more Native females (18.6\%) than Alien $>5$ year $(11.5 \%)$ and Alien $\leq 5$ year $(10.1 \%)$ females ever had coitus on the first date. Significantly more Native females $(11.9 \%)$ than Alien $>5$ year $(6.2 \%)$ and Alien $\leq 5$ year $(7.2 \%)$ females ever had coitus with a male they knew for less than 24 hours. Significantly more Native females (6.4\%) than Alien $>5$ year (1.4\%) and Alien $\leq 5$ year (4.3\%) females ever had simultaneous coitus with one male and one female. Significantly more Native females (32.4\%) than Alien $>5$ year $(22.6 \%)$ and Alien $\leq 5$ year (17.3\%) females ever had interracial coitus (Table 8.2).

Significantly more Native females $(39.6 \%)$ than Alien $>5$ year $(28.8 \%)$ and Alien $\leq 5$ year $(22.3 \%)$ females ever had coitus after consuming alcohol. Significantly more Native females $(28.9 \%)$ than Alien $>5$ year $(11.1 \%)$ and Alien $\leq 5$ year $(7.9 \%)$ females ever had coitus after using marijuana. Significantly more Native females $(4.0 \%)$ than Alien $>5$ year $(2.9 \%)$ and Alien $\leq 5$ year $(0.0 \%)$ females ever had coitus after using cocaine (Table 8.2).

Significantly more Native females $(13.8 \%)$ than Alien $>5$ year $(10.1 \%)$ and Alien $\leq 5$ year (5.0\%) females ever had coitus while bound. Significantly more Native females $(9.1 \%)$ than Alien $>5$ year (5.8\%) and Alien $\leq 5$ year (3.6\%) females ever had coitus while their male partner was bound. Significantly more Native females (29.4\%) than Alien $>5$ year $(25.5 \%)$ and Alien $\leq 5$ year $(11.5 \%)$ females ever had coitus after spanked by the male. Significantly more Native 
females $(13.6 \%)$ than Alien $>5$ year $(11.1 \%)$ and Alien $\leq 5$ year $(2.2 \%)$ females ever had coitus after administering pain to their male partner. Significantly more Native females $(22.5 \%)$ than Alien $>5$ year $(15.9 \%)$ and Alien $\leq 5$ year $(6.5 \%)$ females ever had coitus after receiving pain from their male partner. Significantly more Native females (9.6\%) than Alien>5 year (11.5\%) and Alien $\leq 5$ year $(2.9 \%)$ females ever performed coital dominance over their male partner's submissiveness. Significantly more Native females (50.0\%) than Alien $>5$ year (45.2\%) and Alien $\leq 5$ year $(29.5 \%)$ females ever had a coital orgasm. Significantly more Native females $(34.2 \%)$ than Alien $>5$ year $(26.0 \%)$ and Alien $\leq 5$ year $(16.5 \%)$ females ever faked a coital orgasm (Table 8.2).

Significantly more Native females $(20.1 \%)$ than Alien $>5$ year $(13.0 \%)$ and Alien $\leq 5$ year $(10.1 \%)$ females were photographed or video recorded during coitus. Significantly more Native females $(18.8 \%)$ than Alien $>5$ year $(15.4 \%)$ and Alien $\leq 5$ year $(7.9 \%)$ females ever had a penis penetrate their anus. However, there was no significant difference in females' acculturation exposure and their ever being paid as a prostitute (Table 8.2).

Table 8.2. Female ever-experienced oral-genital and coital practices.

\begin{tabular}{|c|c|c|c|c|c|}
\hline & $\%$ & $n$ & $X^{2}$ & $d f$ & $p$ value \\
\hline Received female performed cunnilingus & & 943 & 21.979 & 2 & .000 \\
\hline Native & 18.3 & & & & \\
\hline Alien $\leq 5 \mathrm{yr}$ & 5.0 & & & & \\
\hline Alien $>5 \mathrm{yr}$ & 9.1 & & & & \\
\hline Received male performed cunnilingus & & 943 & 24.824 & 2 & .000 \\
\hline Native & 71.0 & & & & \\
\hline Alien $\leq 5 \mathrm{yr}$ & 48.9 & & & & \\
\hline Alien $>5 \mathrm{yr}$ & 64.9 & & & & \\
\hline Performed fellatio & & & 14.577 & 2 & .001 \\
\hline Native & 62.1 & 943 & & & \\
\hline Alien $\leq 5 \mathrm{yr}$ & 44.6 & & & & \\
\hline Alien $>5 \mathrm{yr}$ & 56.2 & & & & \\
\hline Performed cunnilingus & & 943 & 13.688 & 2 & .001 \\
\hline Native & 17.3 & & & & \\
\hline Alien $\leq 5 \mathrm{yr}$ & 6.5 & & & & \\
\hline Alien $>5 \mathrm{yr}$ & 10.6 & & & & \\
\hline $\begin{array}{l}\text { Male performed cunnilingus as you performed } \\
\text { fellatio }\end{array}$ & & 943 & 6.538 & 2 & .038 \\
\hline Native & 47.0 & & & & \\
\hline Alien $\leq 5 \mathrm{yr}$ & 35.3 & & & & \\
\hline Alien $>5 \mathrm{yr}$ & 42.8 & & & & \\
\hline Received male performed analingus & & 943 & 14.346 & 2 & .001 \\
\hline
\end{tabular}




\begin{tabular}{|c|c|c|c|c|c|}
\hline & $\%$ & $n$ & $x^{2}$ & $d f$ & $p$ value \\
\hline Native & 27.2 & & & & \\
\hline Alien $\leq 5 \mathrm{yr}$ & 12.9 & & & & \\
\hline Alien $>5 \mathrm{yr}$ & 20.2 & & & & \\
\hline Ever had coitus & & 943 & 21.755 & 2 & .000 \\
\hline Native & 76.3 & & & & \\
\hline Alien $\leq 5 \mathrm{yr}$ & 56.8 & & & & \\
\hline Alien $>5 \mathrm{yr}$ & 73.6 & & & & \\
\hline Ever had coitus on a first date & & 943 & 9,840 & 2 & .007 \\
\hline Native & 18.6 & & & & \\
\hline Alien $\leq 5 \mathrm{yr}$ & 10.1 & & & & \\
\hline Alien $>5 \mathrm{yr}$ & 11.5 & & & & \\
\hline Ever had coitus with partner known for $<24$ hours & & 943 & 6.907 & 2 & .032 \\
\hline Native & 11.9 & & & & \\
\hline Alien $\leq 5 \mathrm{yr}$ & 7.2 & & & & \\
\hline Alien $>5 \mathrm{yr}$ & 6.2 & & & & \\
\hline $\begin{array}{l}\text { Ever simultaneous coitus with one male \& one } \\
\text { female }\end{array}$ & & 943 & 8.078 & 2 & .018 \\
\hline Native & 6.4 & & & & \\
\hline Alien $\leq 5 \mathrm{yr}$ & 4.3 & & & & \\
\hline Alien $>5 \mathrm{yr}$ & 1.4 & & & & \\
\hline Ever had interracial coitus & & 943 & 16.636 & 2 & .000 \\
\hline Native & 32.4 & & & & \\
\hline Alien $\leq 5 \mathrm{yr}$ & 17.3 & & & & \\
\hline Alien $>5 \mathrm{yr}$ & 22.6 & & & & \\
\hline Ever had coitus with alcohol & & 943 & 18.889 & 2 & .000 \\
\hline Native & 39.6 & & & & \\
\hline Alien $\leq 5 \mathrm{yr}$ & 22.3 & & & & \\
\hline Alien $>5 \mathrm{yr}$ & 28.8 & & & & \\
\hline Ever had coitus with marijuana & & 943 & 43.909 & 2 & .000 \\
\hline Native & 28.9 & & & & \\
\hline Alien $\leq 5$ yr & 7.9 & & & & \\
\hline Alien $>5 \mathrm{yr}$ & 11.1 & & & & \\
\hline \multicolumn{6}{|l|}{ Ever had coitus with cocaine } \\
\hline Native & 4.0 & 943 & 6.010 & 2 & .050 \\
\hline
\end{tabular}




\begin{tabular}{|c|c|c|c|c|c|}
\hline & $\%$ & $n$ & $X^{2}$ & $d f$ & $p$ value \\
\hline Alien $\leq 5 \mathrm{yr}$ & 0.0 & & & & \\
\hline Alien $>5 \mathrm{yr}$ & 2.9 & & & & \\
\hline Ever had coitus while bound & & 943 & 8.959 & 2 & .011 \\
\hline Native & 13.8 & & & & \\
\hline Alien $\leq 5 \mathrm{yr}$ & 5.0 & & & & \\
\hline Alien $>5 \mathrm{yr}$ & 10.1 & & & & \\
\hline Ever had coitus while male partner was bound & & 943 & 6.019 & 2 & .049 \\
\hline Native & 9.1 & & & & \\
\hline Alien $\leq 5 \mathrm{yr}$ & 3.6 & & & & \\
\hline Alien $>5 \mathrm{yr}$ & 5.8 & & & & \\
\hline Ever had coitus after you were spanked & & 943 & 18.749 & 2 & .000 \\
\hline Native & 29.4 & & & & \\
\hline Alien $\leq 5 \mathrm{yr}$ & 11.5 & & & & \\
\hline Alien $>5 \mathrm{yr}$ & 25.5 & & & & \\
\hline $\begin{array}{l}\text { Ever had coitus after administering pain to the } \\
\text { male }\end{array}$ & & 943 & 14.667 & 2 & .001 \\
\hline Native & 13.6 & & & & \\
\hline Alien $\leq 5 \mathrm{yr}$ & 2.2 & & & & \\
\hline Alien $>5 \mathrm{yr}$ & 11.1 & & & & \\
\hline Ever had coitus after receiving pain from the male & & 943 & 20.404 & 2 & .000 \\
\hline Native & 22.5 & & & & \\
\hline Alien $\leq 5 \mathrm{yr}$ & 6.5 & & & & \\
\hline Alien $>5 \mathrm{yr}$ & 15.9 & & & & \\
\hline Ever dominant over male's coital submissiveness & & 943 & 8.218 & 2 & .016 \\
\hline Native & 9.6 & & & & \\
\hline Alien $\leq 5 \mathrm{yr}$ & 2.9 & & & & \\
\hline Alien $>5 \mathrm{yr}$ & 11.5 & & & & \\
\hline Ever had a coital orgasm & & 943 & 19.137 & 2 & .000 \\
\hline Native & 50.0 & & & & \\
\hline Alien $\leq 5 \mathrm{yr}$ & 29.5 & & & & \\
\hline Alien $>5 \mathrm{yr}$ & 45.2 & & & & \\
\hline Ever faked a coital orgasm & & 943 & 18.723 & 2 & .000 \\
\hline Native & 34.2 & & & & \\
\hline Alien $\leq 5 \mathrm{yr}$ & 16.5 & & & & \\
\hline
\end{tabular}




\begin{tabular}{lccccc}
\hline & $\boldsymbol{\%}$ & $\boldsymbol{n}$ & $\boldsymbol{X}^{\mathbf{2}}$ & $\boldsymbol{d f}$ & $\boldsymbol{p}$ value \\
\hline Alien $>5$ yr & 26.0 & & & & \\
Ever photographed or videotaped during coitus & & 943 & 11.217 & 2 & .004 \\
$\quad$ Native & 20.1 & & & & \\
Alien $\leq 5$ yr & 10.1 & & & & \\
Alien $>5$ yr & 13.0 & & & \\
Ever had a partner's penis penetrate your anus & & 943 & 9.926 & 2 & .007 \\
$\quad$ Native & 18.8 & & & & \\
Alien $\leq 5$ yr & 7.9 & & & & \\
Alien $>5$ yr & 15.4 & & & \\
Ever paid as a prostitute & & 943 & 1.781 & 2 & .411 \\
Native & 1.6 & & & & \\
Alien $\leq 5$ yr & 2.7 & & & & \\
Alien $>5$ yr & 0.9 & & & &
\end{tabular}

\section{Survival Analysis}

An Acculturative-Exposure survival analysis of 'age at first coitus' and 'age at first coitus on a first date' was calculated for males and females. There were significant differences between Acculturative-Exposure and females 'age at first coitus' $\left(X^{2}(2, \mathrm{~N}=688)=49.827, \mathrm{p}=\right.$ 0.000 ). The trajectory of Native females 'age at first coitus was the youngest throughout the trajectory. Alien $\leq 5$ year females were the oldest, until an intersect with Alien $>5$ year females at age 25.13 years. At that intersect, Alien $>5$ year females were the oldest at first coitus with Alien $\leq 5$ year females trailing towards Native females. This intersect reflected Alien $>5$ year females older age and greater proportion of marrieds than Alien $\leq 5$ year females (Figure 1).

There were significant differences between Acculturative-Exposure and males 'age at first coitus' $\left(X^{2}(2, \mathrm{~N}=432)=14.698, \mathrm{p}=0.001\right)$. The trajectory of Native males 'age at first coitus' was the youngest throughout the trajectory. Alien $\leq 5$ year males were the oldest with two intersects with Alien $>5$ year males at age 23.91 and 33.00 years (Figure 2). 
Figure 1. Females' Age at First Coitus

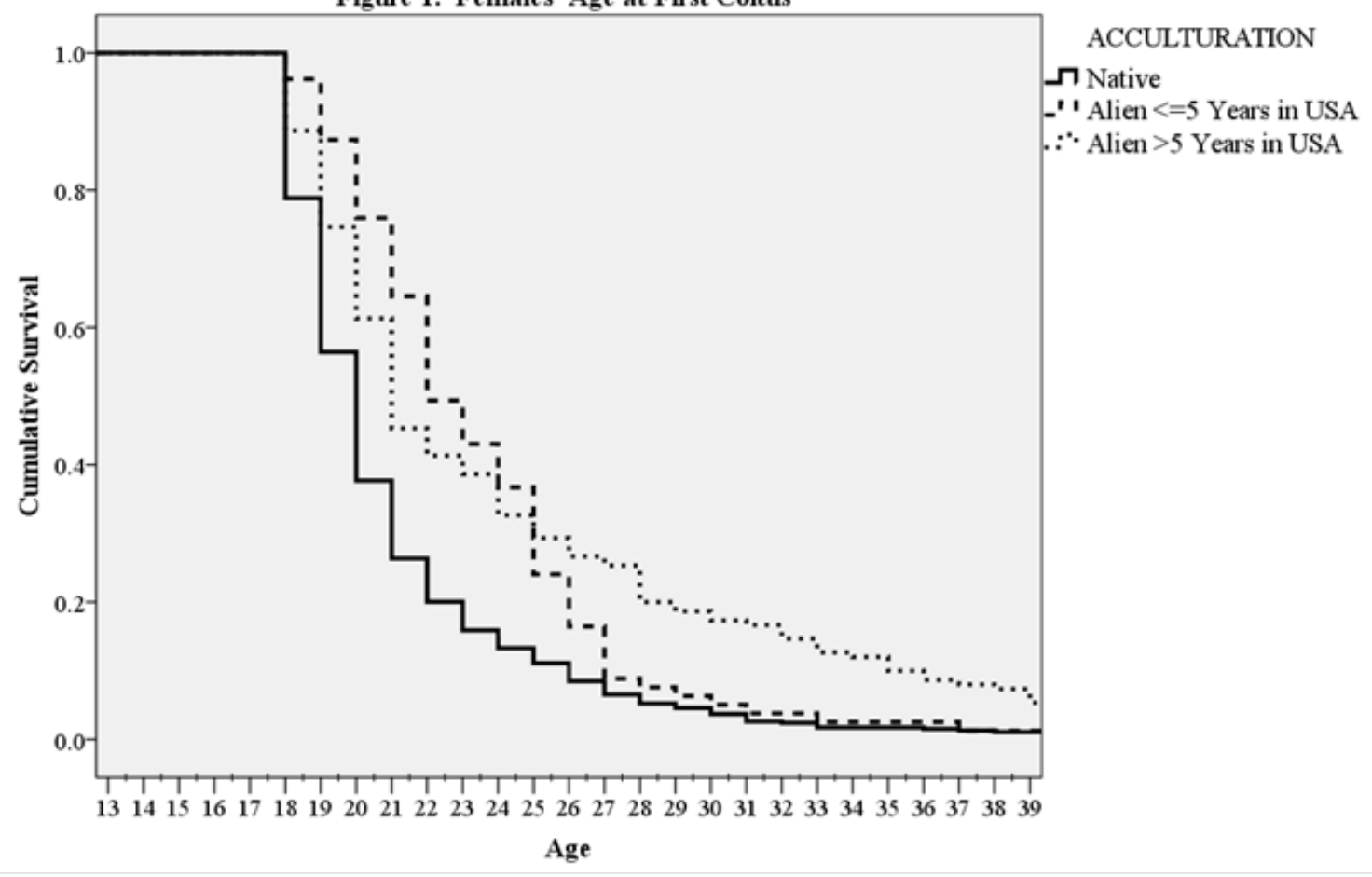

Figure 2. Males' Age at First Coitus

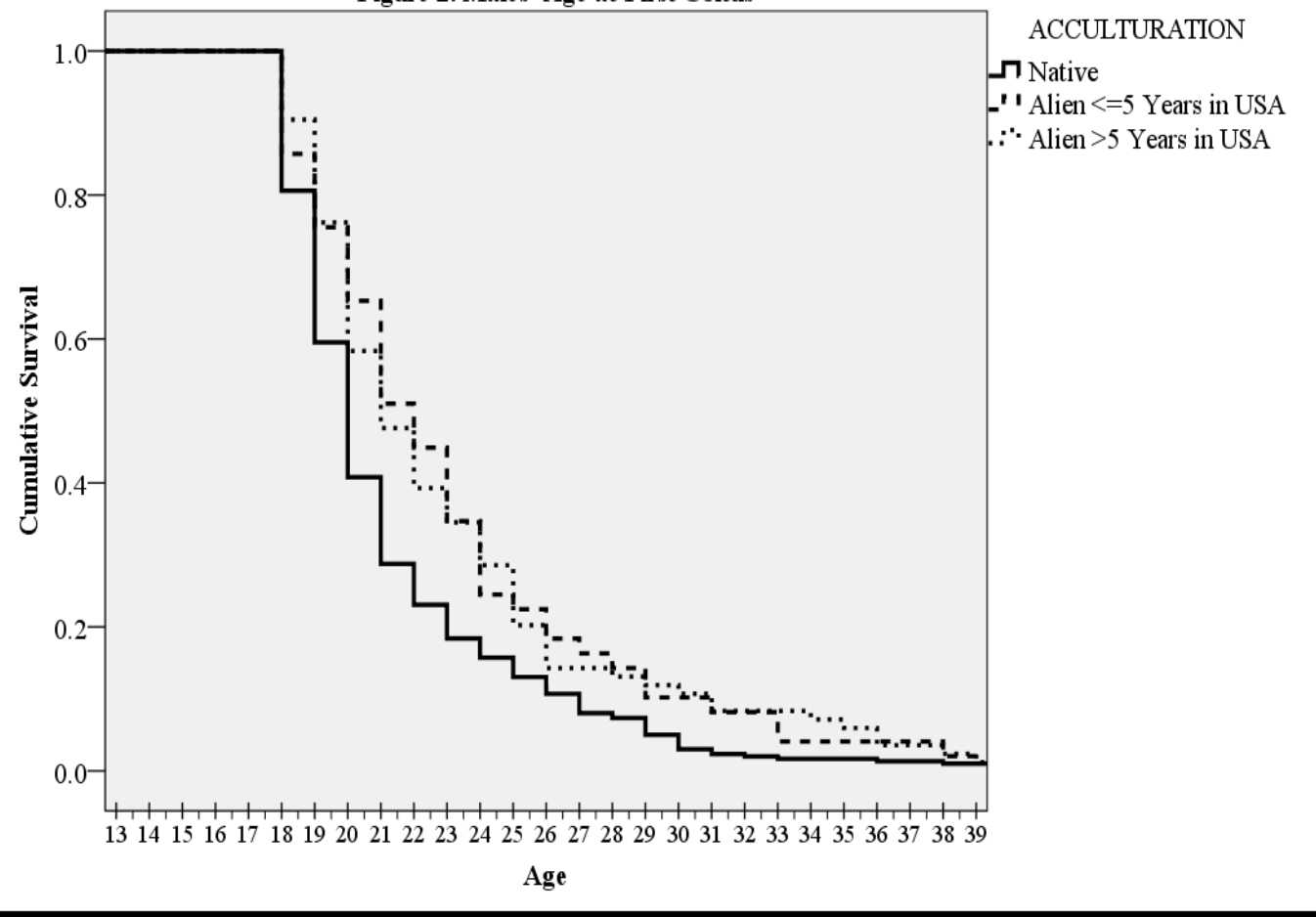


There were significant differences between Acculturative-Exposure and females 'age at first coitus on a first date' $\left(X^{2}(2, \mathrm{~N}=156)=18.491, \mathrm{p}=0.000\right)$. The trajectory of Native females 'age at first coitus on a first date' was the youngest throughout the trajectory. Alien $\leq 5$ year females were the oldest, until an intersect at age 27.13 years. At that intersect Alien $>5$ year females were the oldest at 'first coitus on a first date' with Alien $\leq 5$ year females younger (Figure 3).

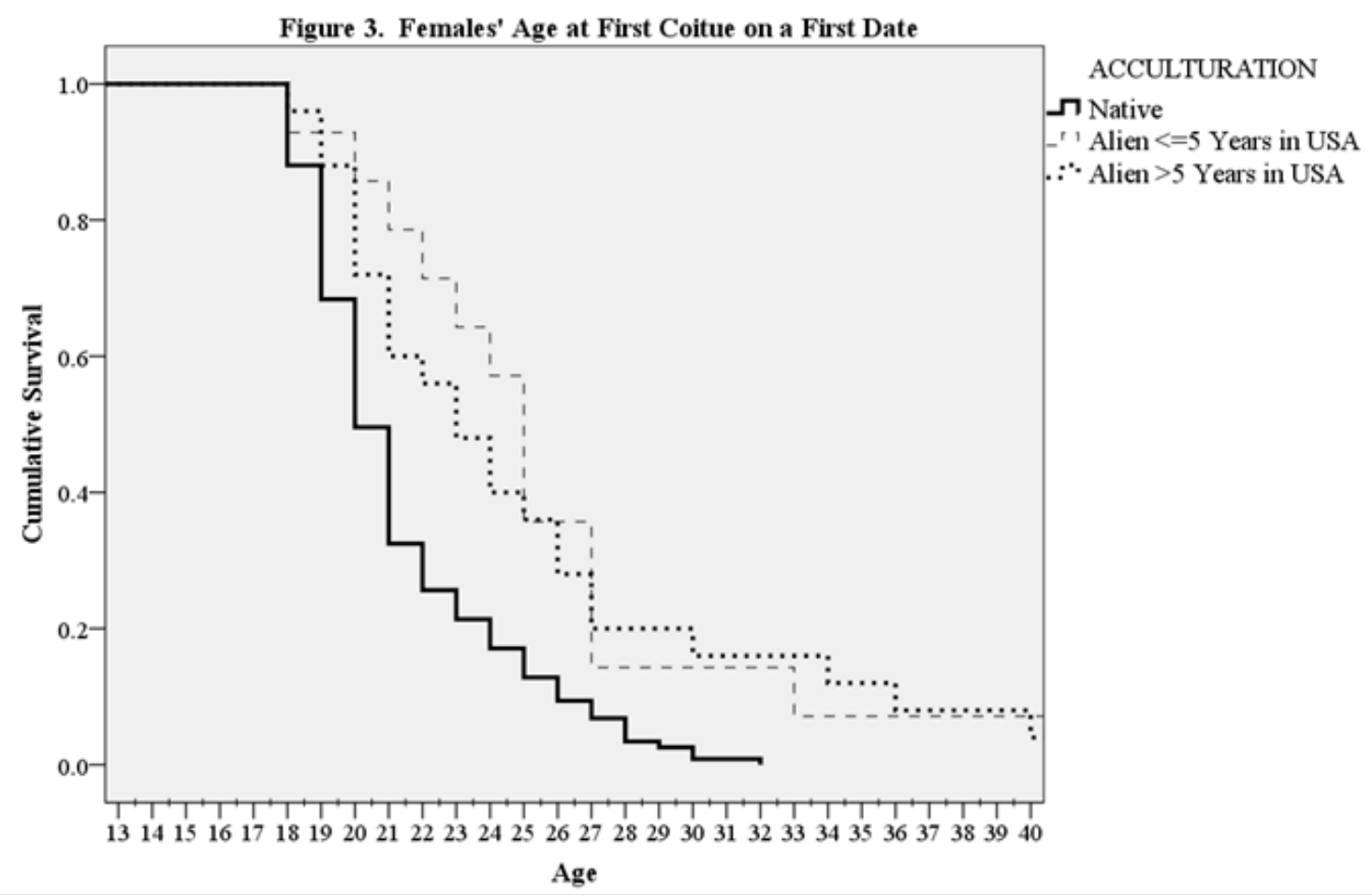

There were significant differences between Acculturative-Exposure and males 'age at first coitus on a first date' $\left(X^{2}(2, \mathrm{~N}=191)=9.766, \mathrm{p}=0.008\right)$. The trajectory of Native males 'age at first coitus on a first date' was the youngest throughout the model. However, Alien $\leq 5$ year and Alien $>5$ year males crisscrossed throughout the trajectory (Figure 4 ). 


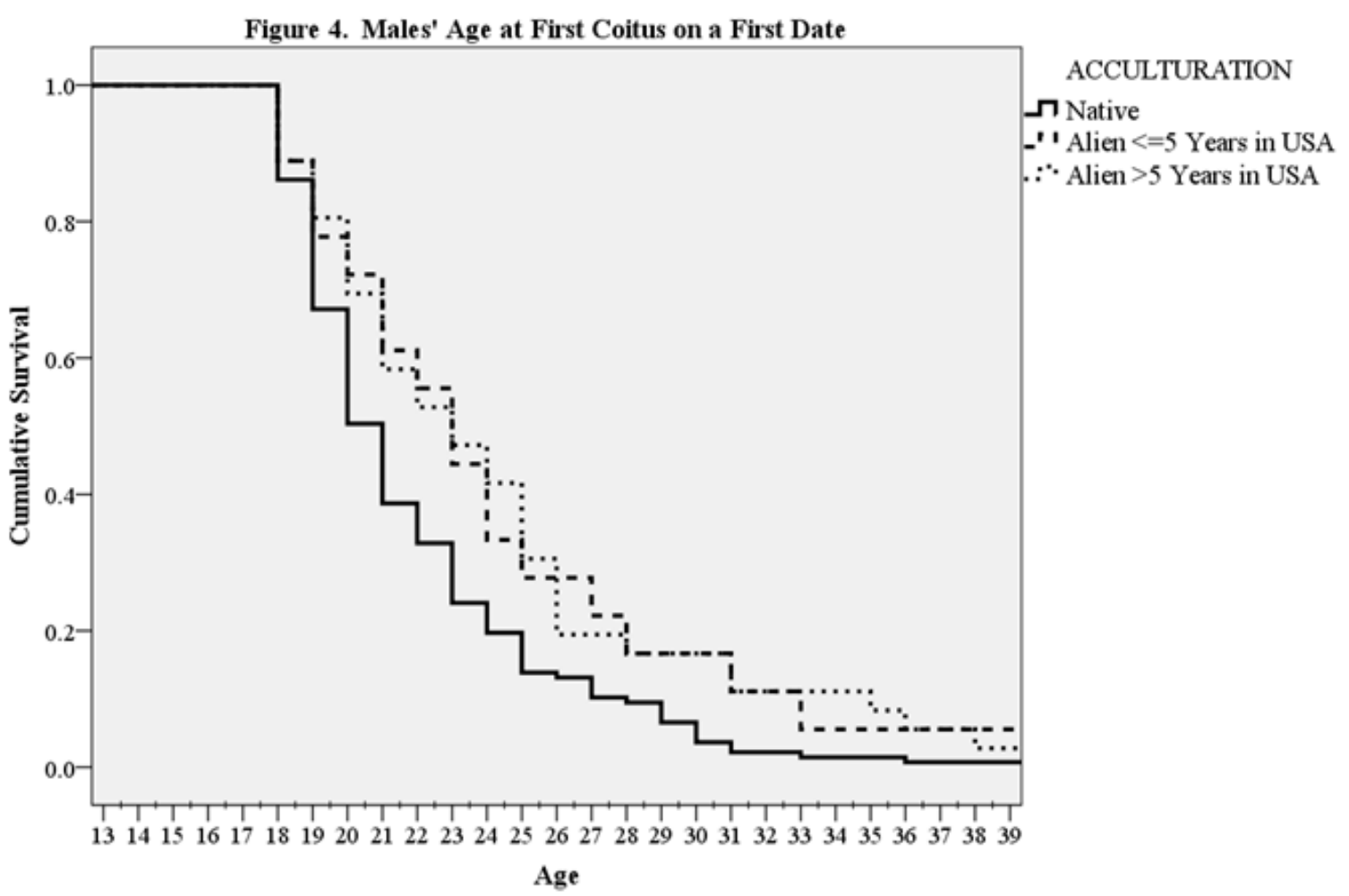

\section{Stepwise Discriminate Analysis}

A stepwise discriminate analysis was conducted to classify males into one of three levels of Acculturative-Exposure. No valid prediction model could be constructed for males. A stepwise discriminate analysis was conducted to classify females into one of three levels of Acculturative-Exposure. No valid prediction model could be constructed between Native females and Alien $>5$ year females.

A valid prediction model was constructed for Native females and Alien $\leq 5$ year females. The predictor variables were; (1) initiation age at male performed cunnilingus, (2) initiation age at performed fellatio, (3) initiation age at male performed cunnilingus while performing fellatio, (4) initiation age at first coitus with a male known for less than 24 hours, (5) initiation age at first interracial coitus, (6) age at first coitus, (7) number of brothers from the same biological parents, (8) number of sisters from the same biological parents, (9) number of lifetime coital partners, and (10) number of coital partners in the last year. The discriminate function yielded a significant association between the group and all predictor variables accounting for $68.9 \%$ of between group variability. The three key structure matrix predictors in the model were; initiation age at male performed cunnilingus (.457), number of brothers from the same biological parents (.376), and initiation age at performing fellatio (.350). The crossvalidated classification yielded an $88.5 \%$ correct classification between Native females and Alien $\leq 5$ year females. 


\section{Limitations}

This investigation should be interpreted with several limitations. Causality cannot be inferred from this cross-sectional analysis. The study used a convenience sample in an ethnically diverse two-year college of undergraduates, limiting its generalizability. However, this can be viewed as a strength since ethnically diverse populations populated with considerable proportions of non-native persons are conspicuously underrepresented or misrepresented in research investigations. In addition, the sample was from a pedestrian undergraduate population not constructed from a preassembled public, private, or political collective identified by their ethnicity, race, immigration status, political affiliation, or public register.

\section{DISCUSSION}

The objective of this study was to describe the consequences of acculturative-exposure on international student populations' core sexual practices, procreation, and sexual orientation.

\section{Males}

The $\mathrm{H}_{1}$ null hypothesis tested for no significant differences between native males and male international students' core masturbatory, oral-genital, or coital practices after five years of exposure to U.S. culture. There was no significant difference in the initiation age at first masturbation for males. Heterosexual male practices of performing cunnilingus, performing cunnilingus while receiving female performed fellatio, and performing analingus on a female were consistent with the immigrant paradox.

There was no significant difference in non-virginity between the male Native and Alien $>5$ year and Alien $\leq 5$ year groups. In the survival analysis of age at first coitus, Native males were consistently younger than Alien $>5$ year and Alien $\leq 5$ year males throughout the trajectory. However, the trajectory lines of Alien $>5$ year and Alien $\leq 5$ year males were undifferentiated while both groups remained older than the Native males throughout the model. This pattern was inconsistent with the immigrant paradox. The survival analysis of 'initiation age at first coitus on a first date' had Native males the youngest throughout the model. However, Alien $\leq 5$ year and Alien $>5$ year males crisscrossed their trajectories throughout the model. This pattern was inconsistent with the immigrant paradox. While 13 of 35 ever-experienced coital practices were consistent with the immigrant paradox, a valid stepwise discriminate analysis prediction model could not be constructed to classify males into the three levels of AcculturativeExposure. We failed to reject the $\mathrm{H}_{1}$ null hypothesis.

The $\mathrm{H}_{2}$ null hypothesis tested for no significant differences between native males and male international students' procreation practices after five years of exposure to U.S. culture. There were no significant differences between Native males and male international students in any of the marital, procreation, or abortion variables tested. We failed to reject the $\mathrm{H}_{2}$ null hypothesis.

$\mathrm{T}_{\mathrm{H}} \mathrm{H}_{3}$ null hypothesis tested for no significant differences between native males and male international students' sexual orientation after five years of exposure to U.S. culture. There were no significant differences between Native males and international student males' sexual orientation after five years of exposure to U.S. culture. Male international students' sexual orientation remained unchanged regardless of years in U.S. residence. We failed to reject the $\mathrm{H}_{3}$ null hypothesis. 
Given the cultural overlap in male sexual practices, procreation, and sexual orientation, it is argued that the observed differences in male international student sexual practices to that of the native U.S. male population may well be attributed to cultural overlap and social opportunity and not the immigrant paradox.

\section{Females}

The $\mathrm{H}_{4}$ null hypothesis tested for no significant differences between native females and female international students' core masturbatory, oral-genital, or coital practices after five years of exposure to U.S. culture. Native females were significantly more experienced than Alien $>5$ year and Alien $\leq 5$ year females in all heterosexual oral-genital practices that is, male performed cunnilingus, performing fellatio, performing fellatio while receiving male performed cunnilingus, and receiving analingus from a male. This was consistent with the immigrant paradox.

Native females had the youngest initiation age at first coitus, with Alien $\leq 5$ year females the oldest, and Alien $>5$ year females in the middle. Native females had the youngest aged partners at their first coitus, with Alien $\leq 5$ year females the oldest, and Alien $>5$ year females in the middle. Native females were significantly more likely to ever had coitus. In the survival analysis of females' age at first coitus, Native females were significantly younger than Alien $>5$ year and Alien $\leq 5$ year females throughout their lifetime. Their trajectory lines held until age 25.13 years at which point the Alien $\leq 5$ year females crossed over the Alien $>5$ year females. Considering that Alien $>5$ year females were significantly older in age than the other two groups, and that Alien $>5$ year females were more likely to be married-these variables were consistent with the immigrant paradox.

Native females had significantly younger initiation ages at 'first interracial coitus' and 'first coitus after using marijuana', with Alien $\leq 5$ year females the oldest and Alien $>5$ year females in the middle. Partners of Native females were younger at first coitus using marijuana, with Alien $\leq 5$ year females' partners the oldest and Alien $>5$ year females' partners in the middle. These coital behaviors were consistent with the immigrant paradox.

'Ever-experienced' female sexual behaviors were consistent with the immigrant paradox with Native females the most experienced, Alien $\leq 5$ year females the least experienced, and Alien $>5$ year females in the middle. The ever-experienced female sexual behaviors were; coitus on a first date, interracial coitus, coitus after consuming alcohol, coitus after using marijuana, coitus after using cocaine, coitus while bound, coitus while male partner was bound, coitus after being spanked, coitus after rendering pain to their male partner, coitus after receiving pain from their male partner, coitus while dominant over their male partner's submissiveness, coitus while being photographed or video recorded, anal penetration by a penis, coital orgasm, and faking coital orgasm. The introductory sexual behaviors of coitus with a male known for less than 24 hours, and simultaneous coitus with one male and one female, had Native females the most experienced, Alien $>5$ year females the least experienced, and Alien $\leq 5$ year females in the middle.

The stepwise discriminate analysis model yielded an $88.5 \%$ cross-validated correct classification between Native females and female international students residing in the U.S. five or less years, which accounted for $68.9 \%$ of between group variability. Given that a prediction model could not discriminate between Native females and Alien $>5$ year females, this analysis supported the immigrant paradox. The $\mathrm{H} 4$ null hypothesis was rejected. 
The $\mathrm{H}_{5}$ null hypothesis tested for no significant differences between native females and female international students' procreation practices after five years of exposure to U.S. culture. Alien $>5$ year females by being older, were more likely to be married and more likely to facilitate a live birth. However, significantly less Alien $\leq 5$ year females than either cohort group were ever impregnated. In addition, significantly more Alien $\leq 5$ year females and their impregnating male partners wanted an abortion than either of the cohort groups. Five times more Alien $\leq 5$ year females $(26.7 \%)$ and Alien $>5$ year females $(28.1 \%)$ were married for their first pregnancy than Native females (5.3\%). Thus, while there were significant procreational differences between Native females and international student females, the direction of the female international students' procreational behaviors were not towards Native females. This suggests that the human underpinning of family and procreation behaviors, regardless of female international students' years in U.S. residence, were not acculturated into those of Native females. With that, the $\mathrm{H}_{5}$ null hypothesis was rejected.

The $\mathrm{H}_{6}$ null hypothesis tested for no significant differences between native females and female international students' sexual orientation after five years of exposure to U.S. culture. The immigrant paradox was consistent for all homosexual oral-genital female practices that is, receiving female performed cunnilingus, and performing cunnilingus. Native females had 1.75 times more homosexuals than Alien $>5$ year females, and 1.86 times more homosexuals than Alien $\leq 5$ year females. Native females had 1.85 times more bisexuals than Alien $>5$ year females, and 4.3 times more bisexuals than Alien $\leq 5$ year females. This shift in sexual orientation was consistent with the immigrant paradox. The $\mathrm{H}_{6}$ null hypothesis was rejected.

The preponderance of female sexual practices including initiation age and introductory female core sexual practices, as well as their sexual orientation, conformed to the immigrant paradox. Female international students' sexual practices and sexual orientation had shifted over time to that of the Native population's sexual practice profile and sexual orientation. The one incongruity to this pattern involved female international students' marital status at first pregnancy. Regardless of years in U.S. residence, female international students' marital status at first pregnancy was not acculturated into that of Native females. In sum, female international students' core oral-genital, and coital practices in terms of initiation age and introductory sexual behaviors as well as their shift in sexual orientation to that of the Native female population, supported the immigrant paradox paradigm.

\section{CONCLUSIONS}

There was no wide-ranging shift in male international students' core masturbatory, oral-genital, coital, or procreation practices in terms of initiation age, introductory sexual behaviors, or sexual orientation to that of the native U.S. male population. It was argued that the observed differences in male international students' sexual practices to that of the native U.S. male population might well be attributed to cultural overlap and social opportunity, and not to the immigrant paradox vis-à-vis acculturation.

With the exception of procreation within marriage, female international students' core sexual practices and sexual orientation shifted over time to the higher risk sexual practices of the U.S. native female population. Female international students' initiation age and scope of heterosexual and homosexual oral-genital, and coital practices as well as their sexual orientation was transitioning to that of the native U.S. female population and was consistent with the immigrant paradox paradigm. 


\section{IMPLICATIONS}

International students were profoundly affected by acculturation, especially in their formative years of adolescence and young adulthood. Male international students' mean age at first coitus was 16.2 years with $65 \%$ of males being non-virgins and one of five males impregnating a female. Female international students' mean age at first coitus was 15.8 years for natives and 16.8 years for international students in U.S. residency for more than five years. Female international students after five years of U.S. residency were 'catching-up' to the high-risk sexual practices of native female sexual practices including; anonymous coitus, coitus on a first date, alcohol/marijuana/cocaine use with coitus, penile-anal penetration, miscarriages, and abortions. The presence of these health risks indicate that proforma U.S. state and local school health education and sex education courses are lacking in breadth and age appropriate delivery. U.S. health and sex education courses applied in the junior year of high school are mostly after the fact for high-risk sexual behavior decision-making. Further, these courses rarely educate on U.S. statutory rape laws, as well as the contraindications and infertility risks of contraceptive and contragestive hormones and devices (e.g. IUDs, Essure ${ }^{\circledR}$, Mifepristone, and Drospirenone). Contemporary native and non-native youth rely extensively on wireless and social media for their health and medical information including STDs/STIs. In turn, U.S. youth are prime targets for commercial vendors and health practitioners aggressively promoting proprietary sex and fertility prescription and over-the-counter (OTC) medications, contraceptive/contragestive devices, and gynecological procedures.

Despite this communally conspicuous need--youth and young adults are increasingly denied formal health and sex education courses taught by certified health educators. State education departments as well as state and city higher education systems throughout the U.S. have reduced, repealed, or reneged on their mandates to provide youth and undergraduates formal classroom health and sex education. By example, in 2014, The City University of New York (approximately 250,000 students) enacted general studies (liberal arts) reforms that all but eliminated required health education credit-bearing courses from their applied science, associate, and baccalaureate degree programs (Belcastro \& Ramsaroop-Hansen, 2017). The prohibition of required health education credit-bearing courses from degree programs in higher education systems disproportionately disadvantages international undergraduates. International undergraduates often arrive from countries or locales that omit health and sex education courses from secondary school curricular requirements. In turn, international undergraduates most in need of health and sex education credit-bearing courses to support their assimilation into the U.S. culture are ill prepared to navigate as well as avoid the negative health consequences of high-risk sexual practices. Accordingly, we recommend that state and local education systems reexamine and expand their commitment of resources, including certified health educators, to underwrite efficient health and sex education for native and culturally diverse student populations. Lastly, additional empirical evidence is required to expose the differing effects of U.S. acculturation on race/ethnicity, natal gender, and sexual orientation.

\section{REFERENCES}

Belcastro, P.A. (1983). A comparison of latent sexual behavior patterns between raped and never raped female victims. Victomology: An International Journal, 7(1-4), 224-230.

Belcastro, P.A. (1985). Sexual behavior differences between black and white students. Journal of Sex Research, 21(1), 56-67. 
Belcastro, P.A., \& Ramsaroop-Hansen, H. (2017). Addressing the antinomy between health education and health literacy in advancing personal health and public health outcomes. Journal of School Health, 87(12), 968-974.

Blake, S. M., Ledsky, R., Goodenow, C., \& O'Donnell, L. (2001). Recency of immigration,

substance use, and sexual behavior among Massachusetts adolescents. American Journal of Public Health, 91(5), 794-798.

Coleman-Minahan, K., Chavez, M., \& Bull, S. (2017). Immigrant generation and sexual initiation among a diverse racial/ethnic group of urban youth. Journal of Immigrant and Minority Health, 9(6), 1412-1419. doi: 10.1007/s10903-016-0420-4

Garcia Coll, C., \& Marks, A. K. (2012). The immigrant paradox in children and adolescents: Is becoming American a developmental risk? Washington D.C.: American Psychological Association.

Du, H., \& Li, X. (2015). Acculturation and HIV-related sexual behaviours among international migrants: A systematic review and meta-analysis. Health Psychology Review, 9(1), 103-122.

Graves, T. D. (1967). Psychological acculturation in a tri-ethnic community. Southwestern Journal, 23, 337-350.

Guarini, T. E., Marks, A. K., Patton, F., \& Garcia Coll, C. (2011). The immigrant paradox in sexual risk behavior among Latino adolescents: Impact of immigrant generation and gender. Applied Developmental Science, 15(4), 201-209.

Guarini, T. E., Marks, A. K., Patton, F., \& García Coll, C. (2013). The immigrant paradox in pregnancy: Explaining the first-generation advantage for Latina adolescents. Journal of Research on Adolescence, 25(1), 14-19.

Guilamo-Ramos, V., Jaccard, J., Pena, J., \& Goldberg, V. (2005). Acculturation-related variables, sexual initiation, and subsequent sexual behavior among Puerto Rican, Mexican, and Cuban youth. Health Psychology, 24(1), 88-95.

Haderxhanaj, L. T., Rhodes, S. D., Romaguera, R. A., Bloom, F. R., \& Leichliter, J. S. (2015). Hispanic men in the U.S.: Acculturation and recent sexual behaviors with female partners, 20062010. American Journal of Public Health, 105(8), E126-E133.

Herbenick D., Bowling J., Fu, T-C., Dodge, B., Guerra-Reyes, L., \& Sanders, S. (2017). Sexual diversity in the U.S.: Results from a nationally representative probability sample of adult women and men. PLoS ONE, 1-23. doi: org/10.1371/journal.pone.0181198

Institute of International Education. (2017). Open doors report on international educational exchange. Retrieved from: https://www.iie.org/en/Research-and-Insights/Open-Doors/Data/InternationalStudents/Leading-Host-Institutions.

Lopez-Gonzalez, L., Aravena, V. C., \& Hummer, R. A. (2005). Immigrant acculturation, gender and health behavior: A research note. Social Forces, 84, 1, 581-593. Retrieved from: http://www.jstor.org/stable/3598318

Marks, A. K., Ejesi, K., \& Garcia Coll, C. (2014). Understanding the U.S. immigrant paradox in childhood and adolescence. Child Development Perspectives, 8(2), 59-64.

Montealegre, J. R., Risser, J. M., Selwyn, B. J., Mccurdy, S. A., \& Sabin, K. (2012). Prevalence of hiv risk behaviors among undocumented central american immigrant women in houston, texas. AIDS and Behavior, 16(6), 1641-1648. Retrieved from: http://dx.doi.org/10.1007/s10461011-0130-9

Parrado, E. A., \& Flippen, C. A. (2010). Migration and sexuality: A comparison of mexicans in sending and receiving communities. Journal of Social Issues, 66(1), 175-195.

Sastre, F., Sanchez, M., \& De La Rosa, M. (2015). Changes in pre-to post-immigration hiv risk behaviors among recent Latino immigrants. AIDS Education and Prevention, 27(1), 44-57.

Schwartz, S. J., Unger, J. B., Des Rosiers, S. E., Lorenzo-Blanco, E. I., Zamboanga, B. L., Huang, S. et al. (2014). Domains of acculturation and their effects on substance use and sexual behavior in recent hispanic immigrant adolescents. Prevention Science, 15(3), 385-396. 
Teruya, S., \& Bazargan-Hejazi, S. (2013). The immigrant and hispanic paradoxes: A systematic review of their predictions and effects. Hispanic Journal of Behavioral Sciences, 35(4), 486-509. doi:10.1177/0739986313499004.

Zhang, X., Rhoads, N., Rangel, M. G., Hovell, M. F., Magis-Rodriguez, C., Sipan, C. L. et al. (2017). Understanding the impact of migration on hiv risk: An analysis of mexican migrants' sexual practices, partners, and contexts by migration phase. AIDS and Behavior, 21(3), 935-948. Retrieved from: http://dx.doi.org/10.1007/s10461-016-1622-4

PHILIP A. BELCASTRO, Ph.D. is a Professor in the Health Education Department at the City University of New York-Borough of Manhattan Community College. His research focuses on the health and wellness of persons as well as school and community populations. E-mail: pbelcastro@bmcc.cuny.edu

HARDAYE RAMSAROOP-HANSEN, EdD, MSW, is an Associate Professor in the Health Education Department at the City University of New York-Borough of Manhattan Community College. Her research focuses on the health and wellness of persons as well as school and community populations. Email: hhansen@bmcc.cuny.edu 